\title{
Meta-analysis of prostate cancer gene expression data identifies a novel discriminatory signature enriched for glycosylating enzymes
}

\author{
Stefan J Barfeld ${ }^{2 *}$, Philip East ${ }^{1}$, Verena Zuber ${ }^{2}$ and Ian G Mills ${ }^{2,3^{*}}$
}

\begin{abstract}
Background: Tumorigenesis is characterised by changes in transcriptional control. Extensive transcript expression data have been acquired over the last decade and used to classify prostate cancers. Prostate cancer is, however, a heterogeneous multifocal cancer and this poses challenges in identifying robust transcript biomarkers.
\end{abstract}

Methods: In this study, we have undertaken a meta-analysis of publicly available transcriptomic data spanning datasets and technologies from the last decade and encompassing laser capture microdissected and macrodissected sample sets.

Results: We identified a 33 gene signature that can discriminate between benign tissue controls and localised prostate cancers irrespective of detection platform or dissection status. These genes were significantly overexpressed in localised prostate cancer versus benign tissue in at least three datasets within the Oncomine Compendium of Expression Array Data. In addition, they were also overexpressed in a recent exon-array dataset as well a prostate cancer RNA-seq dataset generated as part of the The Cancer Genomics Atlas (TCGA) initiative. Biologically, glycosylation was the single enriched process associated with this 33 gene signature, encompassing four glycosylating enzymes. We went on to evaluate the performance of this signature against three individual markers of prostate cancer, v-ets avian erythroblastosis virus E26 oncogene homolog (ERG) expression, prostate specific antigen (PSA) expression and androgen receptor (AR) expression in an additional independent dataset. Our signature had greater discriminatory power than these markers both for localised cancer and metastatic disease relative to benign tissue, or in the case of metastasis, also localised prostate cancer.

Conclusion: In conclusion, robust transcript biomarkers are present within datasets assembled over many years and cohorts and our study provides both examples and a strategy for refining and comparing datasets to obtain additional markers as more data are generated.

Keywords: Transcription, Prostate cancer, Signature

\section{Background}

Alterations in transcriptional programmes are often involved in neoplastic transformation and progression and defining these changes will help to understand the underlying biology of the malignancies. Gene Expression Microarray Analysis and more recently high-throughput RNA sequencing

\footnotetext{
*Correspondence: stefan.barfeld@ncmm.uio.no; ian.mills@ncmm.uio.no ${ }^{2}$ Prostate Cancer Research Group, Centre for Molecular Medicine Norway (NCMM), Nordic EMBL Partnership University of Oslo and Oslo University Hospital, Oslo, Norway

${ }^{3}$ Department of Cancer Prevention and Urology, Institute of Cancer Research and Oslo University Hospital, Oslo, Norway

Full list of author information is available at the end of the article
}

(RNA-seq) are commonly used techniques when trying to acquire an unbiased view of the expression levels of large numbers of genes. In order to define more compact and manageable expression modules that might predict risk or prognosis, various approaches have been used across several studies. These include the identification of consensus profiles across multiple datasets [1] and identifying biologically categorised gene sets with enriched representation of deregulated genes [2,3]. Furthermore, smaller expression modules have also been identified using hierarchical clustering methods to generate clusters containing genes with similar expression profiles across glioblastoma samples [3]. The 
high degree of prostate tissue heterogeneity, however, represents a challenge for transcriptomics since the relative prevalence of each cell type within a given sample determines the overall expression profile. This makes it difficult to compare prostate samples that have very different epithelial and stromal contents. Many studies have compared tumor tissue with benign hyperplastic tissue, or with nontumoral prostate tissues that were not precisely characterised in terms of location or epithelial representation. Therefore, the outcomes of these analyses were possibly biased because the comparisons included tissues of diverse histological or embryological origins. Various approaches have been used to overcome this issue including in silico corrections to compensate for variable epithelial representations in different samples [4], and laser microdissection combined with in vitro linear amplification [5]. The laser capture microdissection study of Tomlins et al. yielded several informative molecular concepts (multi-gene modules), which provide a rich source of data for further refinement and follow-up as well as distinguishing between stromal and epithelial cancer signatures [5]. It is, however, not clear how detectable those concepts might be in material extracted from heterogeneous whole tissue sections, an important point given the time and expense associated with laser capture microdissection.

In this study, we have therefore set out with a number of goals. First and foremost amongst these was to determine whether we could identify gene signatures that were statistically significant in datasets generated from both whole tissue sections and laser capture microdissected material. If so, this might indicate that with the right filtering approach, sample heterogeneity might not be a completely confounding challenge to transcriptomic analysis. Secondly, if we were able to identify such signatures, we then wanted to be able to refine them to a point that the signature and any pathway or process enriched within it could be easily validated by other experimental and clinical research groups. Here, we report a concise 33-gene signature with biological enrichment for glycosylation, which discriminates between benign tissue and prostate cancer ( $\mathrm{PCa}$ ) across multiple transcript detection platforms and sample types.

\section{Methods}

\section{Description of datasets}

Five datasets were downloaded and used in this study.

1. A 19-sample dataset generated by Varambally et al., using the Affymetrix Human Genome U133 Plus 2.0 Array platform. The dataset consisted of 13 macrodissected individual benign prostate, primary and metastatic PCa samples and 6 pooled samples from benign, primary or metastatic PCa tissues. The expression array data were downloaded from GEO under accession number GSE3325 (http://www.ncbi. nlm.nih.gov/geo/query/acc.cgi?acc=GSE3325).

2. A 104-sample dataset generated by Tomlins et al., using an in-house cDNA microarray platform (Chinnaiyan Human 20K Hs6). Laser capture microdissection was used to isolate 101 specific cell populations from 44 individuals representing $\mathrm{PCa}$ progression in a range of sample categories encompassing 12 stromal and 89 epithelial cell populations. These were subcategorised as EPI_BPH (benign prostatic hyperplasia epithelium), EPI_ADI_PCA (normal epithelium adjacent to PCa), EPI_ATR (atrophic epithelium - simple atrophy), EPI_ATR_PIA (atrophic epithelium), PIN (prostatic intraepithelial neoplasia), PCA (prostate carcinoma), MET_HN (Metastatic Prostate Carcinoma - Hormone Naive), MET_HR (Metastatic Prostate Carcinoma Hormone Refractory), STROMA_EPIBPH (BPH Stroma - Epithelial BPH ), STROMA_NOR (Normal Stroma - Organ Donor), STROMA_ADI_PCA (Normal Stroma - Adjacent to prostate cancer). In addition three samples were EPI_NOR (Normal Epithelium - Organ Donor). In our study we maintain this nomenclature in describing the dataset. The expression array data were downloaded from GEO under accession number GSE6099 (http://www.ncbi.nlm.nih.gov/geo/query/ acc.cgi?acc=GSE6099).

3. A multi-cancer microarray dataset generated by Ramaswamy et al., and consisting of 218 tumour samples, spanning 14 common tumour types, and 90 normal tissue samples and profiled on Affymetrix oligonucleotide microarrays (Hu6800 and Hu35KsubA GeneChips). The 14 tumour types incorporated into this study were breast adenocarcinoma, prostate adenocarcinoma, lung adenocarcinoma, colorectal adenocarcinoma, lymphoma, bladder transitional cell carcinoma, melanoma, uterine adenocarcinoma, leukemia, renal cell carcinoma, pancreatic adenocarcinoma, ovarian adenocarcinoma, pleural mesothelioma and cancers of the central nervous system. The dataset was downloaded from the Broad Institute website (http://www.broadinstitute.org/cgi-bin/cancer/ publications/pub_paper.cgi?mode=view\&paper_id=61).

4. A PCa dataset generated by Taylor et al., for 150 tumours, 29 matched normal samples, and 6 cell lines using the Affymetrix Human Exon 1.0 ST array platform. There were 27 metastatic samples amongst the 150 tumours and 35 cases of biochemical relapse (Additional file 1 in Taylor et al.,). The expression array data were downloaded from GEO under accession number GSE21034 (http://www.ncbi.nlm.nih.gov/geo/query/acc.cgi? acc $=$ GSE21034).

5. High throughput RNA sequencing data were generated by the The Cancer Genomics Atlas 
(TCGA) consortium for 383 samples, 50 benign samples and 333 primary tumours. 48 of these samples represented advanced disease with Gleason grade $\geq 8$ and 13 cases had undergone progression as characterised by post-operative biochemical recurrence. Data were downloaded from the UCSC Cancer Genome Browser (https://genome-cancer.ucsc.edu/) TCGA_PRAD_exp_HiSeqV2-2014-05-02.tgz. Associated clinical data were downloaded from the TCGA Data Portal (https://tcga-data.nci.nih.gov/tcga/).

6. A PCa dataset generated by Grasso et al., generated for 28 benign prostate tissue samples, 59 localised $\mathrm{PCa}$ and 35 metastatic PCa was generated on two Agilent microarray platforms (whole genome microarray $(4 x 44 \mathrm{~K}, \mathrm{G} 4112 \mathrm{~F})$ and whole human genome oligo microarray (G4112A ). The expression array data were downloaded from GEO under accession number GSE35988 (http://www.ncbi.nlm. nih.gov/geo/query/acc.cgi?acc=GSE35988).

7. Additional datasets were interrogated for the expression of individual genes within signatures through the Oncomine Compendium of Expression Array data (www.oncomine.org).

\section{Prostate cancer dependent expression changes}

To generate an initial broad progression dependent gene set, we used the prostate progression expression dataset GSE3325 (NCBI GEO database). We quantile normalised probe level intensity values and generated probe set signal estimates using RMA $(1,2)$. We first characterised reporters with a coefficient of variance of less than or equal to 0.05 as uninformative and removed them from further analysis. Reporters having intensities below the $10^{\text {th }}$ quantile (3.91) in more than $75 \%$ of the samples were also removed. We identified progression associated expression changes by linear model. Primary tumour versus benign and metastatic versus primary contrasts were run and differential reporters identified using a 0.01 FDR threshold. Reporters were further filtered selecting those with a differential effect size of greater than or equal to 2 -fold. This resulted in a progression signature set of 4662 reporters, 3021 genes (121 primary, 2900 metastatic, primary $\cap$ metastatic $=102$ ). Signatures derived from this primary dataset were subsequently applied to two additional datasets, one prostate dataset generated by Tomlins et al. [5] in a laser capture microdissection study (GSE6099) and another generated by Ramaswamy et al. [6] and representing multi-tissue primary tumours and metastases (accessible through the Broad Institute data repository: http:// www.broadinstitute.org/cgi-bin/cancer/datasets.cgi).

\section{Identifying correlated gene modules}

We clustered our progression gene set using hierarchical clustering with a Ward agglomerative method designed to minimize intra-cluster variance (hclust, Bioconductor) and a 1 - Pearson correlation coefficient dissimilarity measure. We found this method produced a more highly correlated clustering structure when compared to other methods leading to more compact sub-clusters (Additional file 1). We characterised correlated gene modules by cutting the cluster dendrogram at branch lengths ranging from $\log 10(0.05)$ to $\log 10(3000)$ giving 39 equal intervals across the log scale. We removed clusters containing less than 3 members from further analysis. We selected modules defined at branch lengths of 0.6, 0.8, 1.1, 1.9, 2.5, 4.5, 10.6, 24.7 and 101.6 for further analysis since these gave a broad range of cluster numbers. Since a smaller branch length threshold does not always sub divide a parent module modules can be duplicated at different thresholds. These were removed from further analysis assigning them to the largest branch threshold at which they appears. We assigned Gene Ontology classifications to modules by testing for enrichment at GO nodes using a hyper-geometric distribution and a $0.01 \mathrm{p}$-value threshold. We carried out this analysis at the gene level by translating chip reporter probeset ids to Entrez gene ids. All reporters from the progression signature with assigned Entrez gene ids were used as background. Analysis was carried out using the GoStats package, Bioconductor.

\section{Phenotype dependent transcript module expression changes}

To determine differential regulation of modules within other expression datasets we first identified phenotype dependent expression changes for each sample using an absolute fold change filter of greater than 2 . To generate fold changes against which we could filter each gene was scaled to a baseline intensity value. In the case of dataset GSE3325 each signal intensity from the primary tumour samples was scaled to the corresponding median gene signal intensity across the benign tumour samples. Likewise all metastatic samples were scaled to the median across all primary tumour samples. Prior to mapping modules to dataset GSE6099 we background corrected each sample using a normexp method and print tip loess normalised (normalizeWithinArrays(), Bioconductor). We then scaled PIN samples to EPI_ADI_PCA control samples, PCA samples to PIN, MET_HNF to PCA and MET_HR to PCA. To identify hormone refractory dependent expression changes MET_HR samples were scaled to the median across the non-refractory samples MET_HN. To determine module induction or repression within the scaled samples we tested for enrichment of module genes within the sample associated expression changes using a hypergeometric distribution, $<=0.05 \mathrm{fdr}$. Mapping was achieved across array platforms using NCBI Entrez gene ids. Modules with an intersection of less than 3 were discarded from the analysis. 


\section{Phenotype segregation}

To determine if any of the enriched modules were capable of segregating samples on phenotype we built contingency tables across clinical conditions from each of the data sets (Tomlins [5] and Ramaswamy [6]) for induced and repressed modules and tested for sample enrichment using a Fisher's Exact test. Here we tested each phenotypic group against all others from each data set.

\section{Cluster analysis}

To determine the best clustering method and branch length thresholds to apply to our analysis we clustered our 4662 reporter prostate tumour progression signature (Additional file 2: Table S1) using single, average, complete, Ward's ${ }^{1}$ minimum variance method and mcquitty ${ }^{2}$ agglomerative hierarchical clustering methods along with a divisive method. The hclust function from Bioconductor ${ }^{3}$ was used for the agglomerative techniques and the diana function from the cluster package from Bioconductor was used to run the divisive method. We used the cutree function at branch length thresholds ranging from 0.05 to 2 in increments of 0.05 to derive groups of correlated genes. In the case of the Ward agglomerative method where the branch scales [ it is unclear how Ward calculates its branch lengths, need to find out ] branch length thresholds ranged from $\log _{10}(0.05)$ to $\log _{10}(3000)$ in increments of $\log _{10}(3000 / 0.05) /$ 39. Branch length threshold intervals were chosen to produce a broad range of cluster numbers.

\section{Cluster correlation}

To assess the extent to which genes assigned to clusters are correlated we calculated a within-cluster dissimilarity value for each cluster ${ }^{4}$. This is given by

$$
W C=\frac{1}{K} \sum_{k=1}^{K} \frac{1}{2 \mathrm{~N}_{k}} \sum_{i=1}^{N} \sum_{j=1}^{N} d x_{i}, x_{j}
$$

where $d x_{i}, x_{j}$ is the dissimilarity between genes ${ }_{i}$ and ${ }_{j}$ across all samples, $i, j=1,2, \ldots N$ where $N$ is the total number of cluster members and $k=1,2, \ldots, K$ where $K$ is the total number of clusters. In our case the dissimilarity measure is 1 - Pearson correlation coefficient between 2 genes across all samples. $W(C)$ dissimilarity values across the array of branch length thresholds can be seen plotted against cluster number in Additional file 1: Figure S1. As observed the Ward agglomerative method out performs all other methods producing clusters that are less dissimilar and therefore more highly correlated than those generated from other methods relative to the number of clusters produced. These results provide a justification for using hierarchical clustering with a ward agglomerative method to generate sets of co-regulated genes.

\section{Cluster gene ontology entropy}

To quantify the information content of our clusters from a biological perceptive we assigned GO terms to cluster members. This was achieved by mapping GO terms via reporter entrez gene id assignments using the GO.db annotation package from Bioconductor. To quantify the GO information content of a cluster we calculated Shannon Entropy bit values given by:

$$
H X=-\frac{1}{K} \sum_{k=1}^{K} \sum_{i=1}^{N} p x_{i} \log _{b} p x_{i}
$$

where $x_{i}$ is a cluster associated GO term, $\mathrm{p}\left(x_{i}\right)$ is the probability of choosing $x_{i}$ from all cluster GO terms , $i=1,2, \ldots N$ where $N$ is the total number of unique cluster GO terms, $k=1,2, \ldots K$ where $\mathrm{K}$ is the total number of clusters and $b=2 . H(X)$ bit values for different branch length thresholds can be seen plotted against cluster number for the different clustering techniques in Additional file 3: Figure S2. As observed the Ward clustering method produces clusters with higher GO bit values when compared to other methods. This implies greater uncertainly in the GO term mappings for clusters generated by the ward method thus indicating the production of clusters more GO information rich when compared to other methods. This provides further justification for using hierarchical clustering with a ward agglomerative method to generate sets of co-regulated genes.

\section{Visualization of gene signatures through heatmaps}

For visualization, sample groups were averaged using the mean prior to high level mean and variance normalization using the freely available software J-Express 2012 (http://jexpress.bioinfo.no/site/). Subsequently, both sample groups and genes were hierarchically clustered using complete linkage and Euclidian distance using the freely available software Cluster 3.0 (http://bonsai.hgc.jp/ mdehoon/software/cluster/software.htm). Heatmaps were produced using Java Tree View (http://jtreeview.sourceforge.net/).

\section{Evaluating gene signature specificity and sensitivity}

Testset: Grasso 1 Platform GPL6480

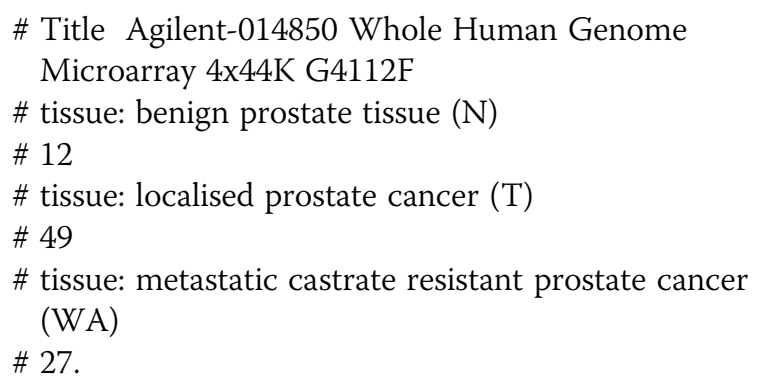


Training data: Grasso 2 Platform GPL6848

\# Title Agilent-012391 Whole Human Genome Oligo Microarray G4112A

\# tissue: benign prostate tissue $(\mathrm{N})$

\# 16

\# tissue: localised prostate cancer $(\mathrm{T})$

\# 10

\# tissue: metastatic castrate resistant prostate cancer (WA) \# 8 .

To evaluate the prediction performance 33 gene signature we analysed data from a microarray experiment of Grasso et al as available from GEO (GSE35988) http:// www.ncbi.nlm.nih.gov/geo/query/acc.cgi?acc=GSE35988.

The dataset includes measurements on two different microarray platforms GPL6480 and GPL6848 and includes three different tissue types (benign, localised and metastatic castrate resistant prostate cancer).

We used the samples typed on platform GPL6848 as trainings data to derive the weights of the genes in the multi-gene signature. First, we replaced missing values using the $\mathrm{k}$ nearest neighbor algorithm as implemented in the R package impute. The gene PCA3 was not measured on the microarray and the gene CRISP3 was not observed in more than half of these samples. Thus, these two genes were excluded from the signature. We estimated the weights of each gene using an L2 regularized logit regression model [7] with the R package glmnet.

Then we used the samples typed on platform GPL6480 as test data to evaluate the prediction performance of the gene-signature. Per sample we computed one score as the weighted average over the 31 proposed genes where the weights were defined by the independent training data. Finally, we computed ROC statistics and report the area under the curve (AUC) of the ROC curve (R package ROCR). The AUC indicates the ability of a marker to distinguish between two groups, where a value 0.5 is random and a value of 1 represents a perfect distinction between the groups. Additionally, we were looking at the AUC of specific genes, in particular KLK3, ERG and AR.

\section{Results and discussion}

In order to define our starting signatures, we selected a dataset published by Varambally et al. about a decade ago and consisting of a small number of whole tissue sections [8]. This constitutes the smallest and oldest dataset used in our meta-analysis. It was, however, extensively validated at both the transcript and protein level in the original study and therefore provides a high-degree of confidence in data quality. We chose to define our starting signatures using this dataset in order to assess how much information could be derived despite the limitations in size and age. Within these data, we firstly identified transcripts that were differentially expressed in localised prostate versus benign tissue or in metastatic disease versus localised cancer using a conventional linear model approach. This approach identified 121 genes differentially expressed in localised primary cancers (primary versus benign, 0.01 FDR) and 2900 genes associated with metastatic status (metastatic versus primary, $0.01 \mathrm{FDR}$ ), which were covered by 4662 probes in total (Additional file 2: Table S1). To further refine these gene lists into discrete signatures, we constructed a gene coexpression network using Pearson correlation coefficients and hierarchical clustering using the Ward agglomerative method (See Methods section).

A number of different correlation or dissimilarity metrics have been employed when constructing co-expression networks. To determine the correlation between the genes we used a Pearson correlation coefficient to construct a dissimilarity matrix across all affected samples in the prostate tumour progression dataset and all genes identified in the preliminary analysis. We then used hierarchical clustering to group the genes. There are a number of available agglomeration methods available each producing their own clustering structure. To determine the best agglomeration method to apply in constructing our expression modules, we clustered our prostate tumour progression signature using single, average, complete, the Ward [9] minimum variance method and the Mcquitty [10] agglomerative hierarchical clustering method along with a divisive method. The performance of these clustering methods by using an algorithm to determine the extent to which genes assigned to clusters are correlated generating a within-cluster dissimilarity value for each cluster (Methods section - 'Cluster Correlation'). In addition, we assessed the information content in gene ontology terms associated with clusters generated using each method by calculating Shannon Entropy bit values (Methods section - 'Cluster Gene Ontology Entropy'). Shannon entropy and coefficient of variation are well known in a great many application domains, from theoretical physics to computational chemistry to materials science. They have been applied in bioinformatics as well, most notably in statistical genetics and molecular biology. Shannon entropy is derived from information theory [11]. Most relevant for this study the approach has previously been used as a measure of the robustness of gene regulatory networks [12], to accelerate feature elimination when classifying microarray expression data [13]. By these measures the Ward clustering method provided both more tightly associated coexpressed gene clusters as well as clusters with higher GO bit values when compared to other methods, indicative of greater information content in the ontologies derived for coexpression clusters generated using the Ward approach than using the other approaches. Additional file 4: Table S2 provides a complete list of coexpressed genes signatures generated used the Ward approach at all branching thresholds. 
Table 1 KEGG pathway enrichment analysis for the genes comprising signature 1 (101.6.1)

\begin{tabular}{|c|c|c|c|c|c|c|c|c|c|c|c|c|}
\hline Category & Term & Count & $\%$ & PValue & Genes & $\begin{array}{l}\text { List } \\
\text { Total }\end{array}$ & $\begin{array}{l}\text { Pop } \\
\text { Hits }\end{array}$ & $\begin{array}{l}\text { Pop } \\
\text { Total }\end{array}$ & $\begin{array}{l}\text { Fold } \\
\text { Enrichment }\end{array}$ & Bonferroni & Benjamini & FDR \\
\hline KEGG_PATHWAY & $\begin{array}{l}\text { hsa04510:Focal } \\
\text { adhesion }\end{array}$ & 37 & 2.86 & 2.01E-06 & $\begin{array}{l}\text { CAV2, CAV1, MYL5, MYL2, TNC, PTEN, MYL9, VCL, IGF1R, LAMB3, } \\
\text { LAMB2, ITGB8, ILK, ITGB6, PDGFC, PAK1, THBS1, THBS4, COL4A4, } \\
\text { PRKCA, ACTB, MET, ITGA1, ACTN1, IGF1, HGF, COL4A6, FLNA, } \\
\text { LAMA4, ITGA6, CCND2, ITGA5, JUN, ITGA8, COL1A2, MYLK, } \\
\text { PTENP1, PARVA }\end{array}$ & 404 & 201 & 5085 & 2.32 & $3.42 \mathrm{E}-04$ & $3.42 \mathrm{E}-04$ & 0 \\
\hline KEGG_PATHWAY & $\begin{array}{l}\text { hsa05414:Dilated } \\
\text { cardiomyopathy }\end{array}$ & 21 & 1.62 & 2.37E-05 & $\begin{array}{l}\text { ACTB, SLC8A1, ACTC1, MYL2, LMNA, ITGA1, IGF1, CACNB2, TPM2, } \\
\text { TPM1, TPM4, TGFB2, DES, ITGA6, ITGA5, ITGB8, PLN, ITGA8, ITGB6, } \\
\text { PRKACB, SGCB }\end{array}$ & 404 & 92 & 5085 & 2.87 & 0 & 0 & 0.03 \\
\hline KEGG_PATHWAY & $\begin{array}{l}\text { hsa05410: } \\
\text { Hypertrophic } \\
\text { cardiomyopathy } \\
\text { (HCM) }\end{array}$ & 20 & 1.55 & $2.50 \mathrm{E}-05$ & $\begin{array}{l}\text { ACTB, SLC8A1, ACTC1, IL6, MYL2, LMNA, ITGA1, IGF1, CACNB2, } \\
\text { TPM2, TPM1, TPM4, TGFB2, DES, ITGA6, ITGA5, ITGB8, ITGA8, } \\
\text { ITGB6, SGCB }\end{array}$ & 404 & 85 & 5085 & 2.96 & 0 & 0 & 0.03 \\
\hline KEGG_PATHWAY & $\begin{array}{l}\text { hsa00280:Valine, } \\
\text { leucine and } \\
\text { isoleucine } \\
\text { degradation }\end{array}$ & 12 & 0.93 & $4.71 \mathrm{E}-04$ & $\begin{array}{l}\text { MCCC2, ALDH7A1, ALDH1B1, MCEE, AOX1, BCKDHB, DLD, } \\
\text { ACAD8, ACAT1, HIBADH, ALDH3A2, AUH }\end{array}$ & 404 & 44 & 5085 & 3.43 & 0.08 & 0.02 & 0.57 \\
\hline KEGG_PATHWAY & $\begin{array}{l}\text { hsa04512:ECM- } \\
\text { receptor interaction }\end{array}$ & 17 & 1.31 & 7.54E-04 & $\begin{array}{l}\text { COL4A4, TNC, ITGA1, COL4A6, CD47, LAMA4, LAMB3, LAMB2, } \\
\text { CD44, ITGA6, ITGB8, ITGA5, ITGA8, ITGB6, COL1A2, THBS1, THBS4 }\end{array}$ & 404 & 84 & 5085 & 2.55 & 0.12 & 0.03 & 0.92 \\
\hline KEGG_PATHWAY & $\begin{array}{l}\text { hsa04270:Vascular } \\
\text { smooth muscle } \\
\text { contraction }\end{array}$ & 20 & 1.55 & 0 & $\begin{array}{l}\text { PRKCA, ACTA2, PPP1R12B, CALD1, MRVI1, KCNMB1, ITPR1, MYL9, } \\
\text { ITPR2, EDNRA, AGTR1, ACTG2, PLCB4, GNAQ, PLA2G12A, MYH11, } \\
\text { PRKACB, PLCB1, PPP1R14A, MYLK }\end{array}$ & 404 & 112 & 5085 & 2.25 & 0.17 & 0.03 & 1.35 \\
\hline KEGG_PATHWAY & $\begin{array}{l}\text { hsa05412: } \\
\text { Arrhythmogenic right } \\
\text { ventricular } \\
\text { cardiomyopathy } \\
\text { (ARVC) }\end{array}$ & 14 & 1.08 & 0.01 & $\begin{array}{l}\text { ACTB, SLC8A1, LMNA, ITGA1, ACTN1, CACNB2, DES, ITGA6, ITGB8, } \\
\text { ITGA5, PKP2, ITGA8, ITGB6, SGCB }\end{array}$ & 404 & 76 & 5085 & 2.32 & 0.65 & 0.14 & 7.2 \\
\hline KEGG_PATHWAY & $\begin{array}{l}\text { hsa04610: } \\
\text { Complement and } \\
\text { coagulation cascades }\end{array}$ & 13 & 1.01 & 0.01 & $\begin{array}{l}\text { C4A, MASP1, C4B, CFB, C1S, CD59, KLKB1, F3, SERPINE1, } \\
\text { SERPINA1, CFI, C2, PROS1 }\end{array}$ & 404 & 69 & 5085 & 2.37 & 0.71 & 0.14 & 8.49 \\
\hline KEGG_PATHWAY & $\begin{array}{l}\text { hsa04310:Wnt } \\
\text { signaling pathway }\end{array}$ & 22 & 1.7 & 0.01 & $\begin{array}{l}\text { PRKCA, CSNK1A1, WNT5B, CAMK2G, MMP7, FZD1, DKK1, PLCB4, } \\
\text { SFRP1, CCND2, SFRP2, JUN, SFRP4, PRICKLE2, PPP3CB, CAMK2D, } \\
\text { WIF1, PRKACB, AXIN2, PLCB1, MYC, APC }\end{array}$ & 404 & 151 & 5085 & 1.83 & 0.72 & 0.13 & 8.79 \\
\hline KEGG_PATHWAY & $\begin{array}{l}\text { hsa05332:Graft- } \\
\text { versus-host disease }\end{array}$ & 9 & 0.7 & 0.01 & $\begin{array}{l}\text { HLA-DQB1, IL6, HLA-DRB1, HLA-DRB4, HLA-C, HLA-DPA1, HLA-B, } \\
\text { FAS, HLA-DMB, HLA-G, HLA-DQA1 }\end{array}$ & 404 & 39 & 5085 & 2.9 & 0.82 & 0.16 & 11.58 \\
\hline KEGG_PATHWAY & $\begin{array}{l}\text { hsa00590:Arachidonic } \\
\text { acid metabolism }\end{array}$ & 11 & 0.85 & 0.01 & $\begin{array}{l}\text { CYP2U1, GPX2, PTGIS, PTGS2, PTGDS, ALOX15B, PLA2G12A, } \\
\text { PTGS1, GGTLC3, GGT1, ALOX5, CBR3 }\end{array}$ & 404 & 56 & 5085 & 2.47 & 0.86 & 0.16 & 13.08 \\
\hline KEGG_PATHWAY & $\begin{array}{l}\text { hsa00480:Glutathione } \\
\text { metabolism }\end{array}$ & 10 & 0.77 & 0.02 & $\begin{array}{l}\text { GSTM1, GPX2, GSTM2, GSTA4, GGTLC3, GSTZ1, GSTO2, ANPEP, } \\
\text { GSTT2, GGT1, GSTO1 }\end{array}$ & 404 & 50 & 5085 & 2.52 & 0.93 & 0.2 & 17.11 \\
\hline KEGG_PATHWAY & $\begin{array}{l}\text { hsa04514:Cell } \\
\text { adhesion molecules } \\
\text { (CAMs) }\end{array}$ & 19 & 1.47 & 0.02 & $\begin{array}{l}\text { HLA-DQB1, HLA-DRB1, CDH1, ITGB2, HLA-C, NEO1, HLA-B, HLA- } \\
\text { DMB, CDH3, HLA-DQA1, HLA-G, ITGA6, ITGB8, ITGA8, PVRL3, CD2, } \\
\text { HLA-DRB4, HLA-DPA1, JAM2, NEGR1, SELE }\end{array}$ & 404 & 132 & 5085 & 1.81 & 0.93 & 0.19 & 17.38 \\
\hline
\end{tabular}


Table 1 KEGG pathway enrichment analysis for the genes comprising signature 1 (101.6.1) (Continued)

\begin{tabular}{|c|c|c|c|c|c|c|c|c|c|c|c|c|}
\hline KEGG_PATHWAY & $\begin{array}{l}\text { hsa04940:Type I } \\
\text { diabetes mellitus }\end{array}$ & 9 & 0.7 & 0.02 & $\begin{array}{l}\text { HLA-DQB1, HLA-DRB1, PTPRN2, HLA-DRB4, HLA-C, HLA-DPA1, } \\
\text { HLA-B, FAS, HLA-DMB, HLA-G, HLA-DQA1 }\end{array}$ & 404 & 42 & 5085 & 2.7 & 0.93 & 0.17 & 17.49 \\
\hline KEGG_PATHWAY & $\begin{array}{l}\text { hsa04350:TGF-beta } \\
\text { signaling pathway }\end{array}$ & 14 & 1.08 & 0.02 & $\begin{array}{l}\text { SMAD6, FST, DCN, TGFB2, ACVR2A, ID1, ZFYVE16, ID4, ID3, } \\
\text { THBS1, MYC, BMPR1A, ACVR1, THBS4 }\end{array}$ & 404 & 87 & 5085 & 2.03 & 0.96 & 0.19 & 20.39 \\
\hline KEGG_PATHWAY & $\begin{array}{l}\text { hsa04810:Regulation } \\
\text { of actin cytoskeleton }\end{array}$ & 27 & 2.09 & 0.02 & $\begin{array}{l}\text { FGFR2, FGF7, MYL5, MYL2, DIAPH2, FGF13, ITGB2, MYL9, VCL, } \\
\text { GSN, ITGB8, ITGB6, RRAS, PDGFC, PAK1, FGF2, APC, ACTB, LIMK2, } \\
\text { ITGA1, ACTN1, ITGA6, CHRM3, ITGA5, CFL2, ITGA8, MYLK }\end{array}$ & 404 & 215 & 5085 & 1.58 & 0.96 & 0.18 & 20.54 \\
\hline KEGG_PATHWAY & $\begin{array}{l}\text { hsa05330:Allograft } \\
\text { rejection }\end{array}$ & 8 & 0.62 & 0.02 & $\begin{array}{l}\text { HLA-DQB1, HLA-DRB1, HLA-DRB4, HLA-C, HLA-DPA1, HLA-B, FAS, } \\
\text { HLA-DMB, HLA-G, HLA-DQA1 }\end{array}$ & 404 & 36 & 5085 & 2.8 & 0.97 & 0.19 & 22.63 \\
\hline KEGG_PATHWAY & $\begin{array}{l}\text { hsa00330:Arginine } \\
\text { and proline } \\
\text { metabolism }\end{array}$ & 10 & 0.77 & 0.02 & $\begin{array}{l}\text { ALDH7A1, ALDH18A1, ACY1, GATM, GLUD2, ALDH1B1, MAOB, } \\
\text { OAT, ALDH3A2, CKB }\end{array}$ & 404 & 53 & 5085 & 2.37 & 0.98 & 0.19 & 23.67 \\
\hline KEGG_PATHWAY & $\begin{array}{l}\text { hsa00982:Drug } \\
\text { metabolism }\end{array}$ & 11 & 0.85 & 0.02 & $\begin{array}{l}\text { GSTM1, GSTM2, CYP3A5, GSTA4, AOX1, MAOB, ADH5, GSTZ1, } \\
\text { GSTO2, GSTT2, GSTO1 }\end{array}$ & 404 & 62 & 5085 & 2.23 & 0.98 & 0.18 & 24.32 \\
\hline KEGG_PATHWAY & hsa05218:Melanoma & 12 & 0.93 & 0.02 & $\begin{array}{l}\text { FGF7, MET, IGF1, CDH1, CDK6, FGF13, HGF, RB1, PTEN, IGF1R, } \\
\text { PDGFC, FGF2, PTENP1 }\end{array}$ & 404 & 71 & 5085 & 2.13 & 0.98 & 0.18 & 24.47 \\
\hline KEGG_PATHWAY & $\begin{array}{l}\text { hsa05416:Viral } \\
\text { myocarditis }\end{array}$ & 12 & 0.93 & 0.02 & $\begin{array}{l}\text { ACTB, HLA-DQB1, CAV1, HLA-DRB1, ITGB2, HLA-C, HLA-B, HLA- } \\
\text { DMB, HLA-DQA1, HLA-G, MYH11, HLA-DRB4, HLA-DPA1, SGCB }\end{array}$ & 404 & 71 & 5085 & 2.13 & 0.98 & 0.18 & 24.47 \\
\hline KEGG_PATHWAY & hsa05310:Asthma & 7 & 0.54 & 0.02 & $\begin{array}{l}\text { FCER1A, HLA-DQB1, HLA-DRB1, HLA-DRB4, FCER1G, HLA-DPA1, } \\
\text { HLA-DMB, HLA-DQA1 }\end{array}$ & 404 & 29 & 5085 & 3.04 & 0.98 & 0.18 & 25.37 \\
\hline KEGG_PATHWAY & $\begin{array}{l}\text { hsa00640:Propanoate } \\
\text { metabolism }\end{array}$ & 7 & 0.54 & 0.04 & ALDH7A1, ALDH1B1, MCEE, SUCLA2, ACAT1, ACSS3, ALDH3A2 & 404 & 32 & 5085 & 2.75 & 1 & 0.25 & 36.89 \\
\hline KEGG_PATHWAY & $\begin{array}{l}\text { hsa04916: } \\
\text { Melanogenesis }\end{array}$ & 14 & 1.08 & 0.05 & $\begin{array}{l}\text { PRKCA, WNT5B, GNAI1, CAMK2G, CREB1, EDN1, FZD1, EDNRB, } \\
\text { PLCB4, GNAQ, CAMK2D, CREB3L4, PRKACB, PLCB1 }\end{array}$ & 404 & 99 & 5085 & 1.78 & 1 & 0.3 & 44.76 \\
\hline KEGG_PATHWAY & $\begin{array}{l}\text { hsa04020:Calcium } \\
\text { signaling pathway }\end{array}$ & 21 & 1.62 & 0.06 & $\begin{array}{l}\text { PRKCA, SLC8A1, CAMK2G, PHKA1, PTGFR, ITPR1, ITPR2, EDNRA, } \\
\text { AGTR1, EDNRB, GNAL, CD38, PLCB4, GNAQ, CHRM3, PLN, } \\
\text { CAMK2D, PPP3CB, PRKACB, PLCB1, MYLK }\end{array}$ & 404 & 176 & 5085 & 1.5 & 1 & 0.37 & 54.64 \\
\hline KEGG_PATHWAY & $\begin{array}{l}\text { hsa04530:Tight } \\
\text { junction }\end{array}$ & 17 & 1.31 & 0.06 & $\begin{array}{l}\text { PRKCA, ACTB, RAB3B, MYL5, MAGI2, ZAK, MYL2, MPDZ, GNAI1, } \\
\text { ACTN1, AMOTL1, PTEN, MYL9, EPB41L2, MYH11, RRAS, JAM2, } \\
\text { PTENP1 }\end{array}$ & 404 & 134 & 5085 & 1.6 & 1 & 0.36 & 54.73 \\
\hline KEGG_PATHWAY & $\begin{array}{l}\text { hsa05222:Small cell } \\
\text { lung cancer }\end{array}$ & 12 & 0.93 & 0.07 & $\begin{array}{l}\text { COL4A4, PTGS2, CDK6, RB1, PTEN, COL4A6, LAMB3, LAMA4, } \\
\text { LAMB2, ITGA6, PIAS1, MYC, PTENP1 }\end{array}$ & 404 & 84 & 5085 & 1.8 & 1 & 0.36 & 56.55 \\
\hline KEGG_PATHWAY & $\begin{array}{l}\text { hsa04360:Axon } \\
\text { guidance }\end{array}$ & 16 & 1.24 & 0.08 & $\begin{array}{l}\text { LIMK2, GNAI1, MET, NTN4, SLIT2, EPHA3, SEMA5A, EPHA4, EPHB6, } \\
\text { CFL2, PPP3CB, SEMA3C, EFNA5, UNC5D, PAK1, RASA1 }\end{array}$ & 404 & 129 & 5085 & 1.56 & 1 & 0.42 & 65.75 \\
\hline KEGG_PATHWAY & $\begin{array}{l}\text { hsa04115:p53 } \\
\text { signaling pathway }\end{array}$ & 10 & 0.77 & 0.09 & $\begin{array}{l}\text { SERPINB5, CCND2, SERPINE1, IGF1, CDK6, FAS, GADD45B, THBS1, } \\
\text { CCNG2, PTEN, PTENP1 }\end{array}$ & 404 & 68 & 5085 & 1.85 & 1 & 0.42 & 66.77 \\
\hline KEGG_PATHWAY & $\begin{array}{l}\text { hsa04720:Long-term } \\
\text { potentiation }\end{array}$ & 10 & 0.77 & 0.09 & $\begin{array}{l}\text { PRKCA, PLCB4, GNAQ, CAMK2G, CAMK2D, PPP3CB, PRKACB, } \\
\text { PLCB1, ITPR1, ITPR2 }\end{array}$ & 404 & 68 & 5085 & 1.85 & 1 & 0.42 & 66.77 \\
\hline KEGG_PATHWAY & $\begin{array}{l}\text { hsa04672:Intestinal } \\
\text { immune network for } \\
\text { IgA production }\end{array}$ & 8 & 0.62 & 0.09 & $\begin{array}{l}\text { HLA-DQB1, IL6, TNFSF13B, HLA-DRB1, HLA-DRB4, HLA-DPA1, } \\
\text { HLA-DMB, HLA-DQA1, TGFB2 }\end{array}$ & 404 & 49 & 5085 & 2.05 & 1 & 0.42 & 68.02 \\
\hline
\end{tabular}


Table 1 KEGG pathway enrichment analysis for the genes comprising signature 1 (101.6.1) (Continued)

\begin{tabular}{|c|c|c|c|c|c|c|c|c|c|c|c|c|}
\hline KEGG_PATHWAY & $\begin{array}{l}\text { hsa05322:Systemic } \\
\text { lupus erythematosus }\end{array}$ & 13 & 1.01 & 0.09 & $\begin{array}{l}\text { HLA-DQB1, HLA-DRB1, C4A, C4B, ACTN1, SSB, C1S, H2AFJ, HLA- } \\
\text { DMB, HLA-DQA1, HLA-DRB4, HLA-DPA1, H3F3B, C2 }\end{array}$ & 404 & 99 & 5085 & 1.65 & 1 & 0.41 & 68.42 \\
\hline KEGG_PATHWAY & $\begin{array}{l}\text { hsa00620:Pyruvate } \\
\text { metabolism }\end{array}$ & 7 & 0.54 & 0.09 & ALDH7A1, ALDH1B1, DLD, ACYP2, DLAT, ACAT1, ALDH3A2 & 404 & 40 & 5085 & 2.2 & 1 & 0.41 & 69.45 \\
\hline KEGG_PATHWAY & $\begin{array}{l}\text { hsa04730:Long-term } \\
\text { depression }\end{array}$ & 10 & 0.77 & 0.09 & $\begin{array}{l}\text { PRKCA, IGF1R, PLCB4, GNAQ, GNAI1, PLA2G12A, IGF1, PLCB1, } \\
\text { ITPR1, ITPR2 }\end{array}$ & 404 & 69 & 5085 & 1.82 & 1 & 0.4 & 69.5 \\
\hline KEGG_PATHWAY & $\begin{array}{l}\text { hsa00980:Metabolism } \\
\text { of xenobiotics by } \\
\text { cytochrome P450 }\end{array}$ & 9 & 0.7 & 0.1 & $\begin{array}{l}\text { GSTM1, GSTM2, CYP3A5, GSTA4, ADH5, GSTZ1, GSTO2, GSTT2, } \\
\text { GSTO1 }\end{array}$ & 404 & 60 & 5085 & 1.89 & 1 & 0.42 & 71.98 \\
\hline
\end{tabular}

Genes comprising signature 1 (Additional file 5: Table S3) were uploaded into the DAVID gene ontology search engine (http://david.abcc.ncifcrf.gov/). KEGG pathway enrichment was generated and the table represents the output file ranked based on significance and annotated by column header. 
Table 2 KEGG pathway enrichment analysis for the genes comprising signature 2 (101.6.2)

\begin{tabular}{|c|c|c|c|c|c|c|c|c|c|c|c|c|}
\hline Category & Term & Count & $\%$ & PValue & Genes & $\begin{array}{l}\text { List } \\
\text { Total }\end{array}$ & $\begin{array}{l}\text { Pop } \\
\text { Hits }\end{array}$ & $\begin{array}{l}\text { Pop } \\
\text { Total }\end{array}$ & $\begin{array}{l}\text { Fold } \\
\text { Enrichment }\end{array}$ & Bonferroni & Benjamini & FDR \\
\hline KEGG_PATHWAY & $\begin{array}{l}\text { hsa04610:Complement and } \\
\text { coagulation cascades }\end{array}$ & 10 & 1.56 & 0 & $\begin{array}{l}\text { C1QA, FGG, A2M, C3, KLKB1, CD46, C1R, SERPING1, C1S, } \\
\text { CFD }\end{array}$ & 219 & 69 & 5085 & 3.37 & 0.33 & 0.33 & 2.97 \\
\hline KEGG_PATHWAY & hsa04540:Gap junction & 10 & 1.56 & 0.01 & $\begin{array}{l}\text { TJP1, ADCY2, GNAI1, PDGFA, TUBB6, GUCY1A3, GJA1, } \\
\text { LPAR1, PRKACB, ITPR2 }\end{array}$ & 219 & 89 & 5085 & 2.61 & 0.88 & 0.65 & 14.98 \\
\hline KEGG_PATHWAY & hsa04142:Lysosome & 11 & 1.72 & 0.03 & $\begin{array}{l}\text { AGA, HGSNAT, LAMP2, CTSK, GM2A, PSAP, LGMN, CTSB, } \\
\text { SCARB2, FUCA1, CLN5 }\end{array}$ & 219 & 117 & 5085 & 2.18 & 0.99 & 0.77 & 28.69 \\
\hline KEGG_PATHWAY & $\begin{array}{l}\text { hsa04270:Vascular smooth } \\
\text { muscle contraction }\end{array}$ & 10 & 1.56 & 0.05 & $\begin{array}{l}\text { PLA2G4A, ADCY2, CALD1, MRVI1, GUCY1A3, PRKCH, } \\
\text { PRKACB, PPP1CB, MYLK, ITPR2 }\end{array}$ & 219 & 112 & 5085 & 2.07 & 1 & 0.87 & 46.04 \\
\hline KEGG_PATHWAY & $\begin{array}{l}\text { hsa04310:Wnt signaling } \\
\text { pathway }\end{array}$ & 12 & 1.88 & 0.06 & $\begin{array}{l}\text { CCND1, PRICKLE1, CCND2, BTRC, NFAT5, CAMK2D, TP53, } \\
\text { MAPK10, PRKACB, FZD5, FZD4, FZD7 }\end{array}$ & 219 & 151 & 5085 & 1.85 & 1 & 0.85 & 51.51 \\
\hline KEGG_PATHWAY & hsa05330:Allograft rejection & 5 & 0.78 & 0.07 & HLA-DRB5, HLA-DPB1, HLA-E, HLA-DOA, HLA-DRA & 219 & 36 & 5085 & 3.22 & 1 & 0.84 & 56.25 \\
\hline KEGG_PATHWAY & hsa05416:Viral myocarditis & 7 & 1.1 & 0.08 & $\begin{array}{l}\text { CAV1, CCND1, HLA-DRB5, HLA-DPB1, HLA-E, HLA-DOA, } \\
\text { HLA-DRA }\end{array}$ & 219 & 71 & 5085 & 2.29 & 1 & 0.86 & 64.57 \\
\hline KEGG_PATHWAY & $\begin{array}{l}\text { hsa05332:Graft-versus-host } \\
\text { disease }\end{array}$ & 5 & 0.78 & 0.08 & HLA-DRB5, HLA-DPB1, HLA-E, HLA-DOA, HLA-DRA & 219 & 39 & 5085 & 2.98 & 1 & 0.82 & 65.24 \\
\hline KEGG_PATHWAY & hsa04510:Focal adhesion & 14 & 2.19 & 0.09 & $\begin{array}{l}\text { CAV1, PDGFA, MAPK10, FLNC, PPP1CB, VCL, CCND1, } \\
\text { CCND2, ITGAV, COL6A2, RAP1A, THBS1, PIK3R1, MYLK }\end{array}$ & 219 & 201 & 5085 & 1.62 & 1 & 0.81 & 67.5 \\
\hline
\end{tabular}

Genes comprising signature 2 (Additional file 6: Table S4) were uploaded into the DAVID gene ontology search engine (http://david.abcc.ncifcrf.gov/). KEGG pathway enrichment was generated and the table represents the output file ranked based on significance and annotated by column header. 
Table 3 KEGG pathway enrichment analysis for the genes comprising signature 3 (101.6.3)

\begin{tabular}{|c|c|c|c|c|c|c|c|c|c|c|c|c|}
\hline Category & Term & Count & $\%$ & PValue & Genes & $\begin{array}{l}\text { List } \\
\text { Total }\end{array}$ & $\begin{array}{l}\text { Pop } \\
\text { Hits }\end{array}$ & $\begin{array}{l}\text { Pop } \\
\text { Total }\end{array}$ & $\begin{array}{l}\text { Fold } \\
\text { Enrichment }\end{array}$ & Bonferroni & Benjamini & FDR \\
\hline KEGG_PATHWAY & $\begin{array}{l}\text { hsa04110:Cell } \\
\text { cycle }\end{array}$ & 36 & 0.47 & 9.03E-20 & $\begin{array}{l}\text { E2F1, E2F2, E2F3, TTK, CHEK1, PTTG1, CCNE2, CCNE1, CDKN2A, } \\
\text { MCM7, CDKN2C, CDKN2D, ORC6L, TFDP2, BUB1, CCNA2, STAG1, } \\
\text { CDC7, CDC6, RBL1, SKP2, ESPL1, CDC20, MCM2, CDC25C, MCM4, } \\
\text { CDC25A, CDC25B, CDKN1C, CCNB1, CCNB2, MAD2L1, PLK1, } \\
\text { GSK3B, BUB1B, MAD2L2 }\end{array}$ & 225 & 125 & 5085 & 6.51 & $1.29 \mathrm{E}-17$ & 1.29E-17 & 1.07E-16 \\
\hline KEGG_PATHWAY & $\begin{array}{l}\text { hsa03030:DNA } \\
\text { replication }\end{array}$ & 12 & 0.16 & $2.15 \mathrm{E}-07$ & $\begin{array}{l}\text { RFC5, PRIM1, MCM7, RFC4, POLE2, LIG1, POLA1, POLA2, MCM2, } \\
\text { RNASEH2A, MCM4, FEN1 }\end{array}$ & 225 & 36 & 5085 & 7.53 & $3.08 \mathrm{E}-05$ & $1.54 \mathrm{E}-05$ & $2.55 E-04$ \\
\hline KEGG_PATHWAY & $\begin{array}{l}\text { hsa04114:Oocyte } \\
\text { meiosis }\end{array}$ & 18 & 0.23 & $4.82 \mathrm{E}-06$ & $\begin{array}{l}\text { SGOL1, AURKA, CDC20, ESPL1, PTTG1, CDC25C, CCNE2, CCNB1, } \\
\text { CCNE1, CCNB2, MAD2L1, ADCY9, CALML3, PLK1, BUB1, FBXO5, } \\
\text { CAMK2B, MAD2L2 }\end{array}$ & 225 & 110 & 5085 & 3.7 & $6.89 \mathrm{E}-04$ & $2.30 \mathrm{E}-04$ & 0.01 \\
\hline KEGG_PATHWAY & $\begin{array}{l}\text { hsa04914: } \\
\text { Progesterone- } \\
\text { mediated oocyte } \\
\text { maturation }\end{array}$ & 14 & 0.18 & $8.26 \mathrm{E}-05$ & $\begin{array}{l}\text { HSP90AA1, CDC25C, CDC25A, CDC25B, CCNB1, CCNB2, MAD2L1, } \\
\text { KRAS, ADCY9, PLK1, BUB1, MAD2L2, PIK3R3, CCNA2 }\end{array}$ & 225 & 86 & 5085 & 3.68 & 0.01 & 0 & 0.1 \\
\hline KEGG_PATHWAY & $\begin{array}{l}\text { hsa04115:p53 } \\
\text { signaling } \\
\text { pathway }\end{array}$ & 10 & 0.13 & 0 & $\begin{array}{l}\text { CCNE2, CCNB1, CCNE1, CDKN2A, CCNB2, RRM2, TSC2, CHEK1, } \\
\text { PMAIP1, GTSE1 }\end{array}$ & 225 & 68 & 5085 & 3.32 & 0.32 & 0.07 & 3.16 \\
\hline KEGG_PATHWAY & $\begin{array}{l}\text { hsa05222:Small } \\
\text { cell lung cancer }\end{array}$ & 11 & 0.14 & 0 & $\begin{array}{l}\text { E2F1, CCNE2, E2F2, CCNE1, CKS1B, E2F3, PTK2, SKP2, PIAS2, } \\
\text { PIK3R3, ITGA2B }\end{array}$ & 225 & 84 & 5085 & 2.96 & 0.4 & 0.08 & 4.12 \\
\hline KEGG_PATHWAY & $\begin{array}{l}\text { hsa04360:Axon } \\
\text { guidance }\end{array}$ & 14 & 0.18 & 0 & $\begin{array}{l}\text { PLXNA1, EFNB3, PLXNA2, DPYSL5, EPHB1, PTK2, KRAS, UNC5B, } \\
\text { PAK2, UNC5A, FYN, GSK3B, SRGAP1, SRGAP2 }\end{array}$ & 225 & 129 & 5085 & 2.45 & 0.45 & 0.08 & 4.83 \\
\hline KEGG_PATHWAY & $\begin{array}{l}\text { hsa00240: } \\
\text { Pyrimidine } \\
\text { metabolism }\end{array}$ & 11 & 0.14 & 0.01 & $\begin{array}{l}\text { PRIM1, TYMS, POLR3K, POLE2, RRM2, RRM1, DCK, POLA1, POLA2, } \\
\text { NME7, TK1 }\end{array}$ & 225 & 95 & 5085 & 2.62 & 0.71 & 0.14 & 9.63 \\
\hline KEGG_PATHWAY & $\begin{array}{l}\text { hsa05219:Bladder } \\
\text { cancer }\end{array}$ & 7 & 0.09 & 0.01 & E2F1, RPS6KA5, E2F2, E2F3, CDKN2A, KRAS, PGF & 225 & 42 & 5085 & 3.77 & 0.74 & 0.14 & 10.67 \\
\hline KEGG_PATHWAY & $\begin{array}{l}\text { hsa05215:Prostate } \\
\text { cancer }\end{array}$ & 10 & 0.13 & 0.02 & $\begin{array}{l}\text { E2F1, CCNE2, E2F2, CCNE1, E2F3, HSP90AA1, KRAS, GSK3B, PIK3R3, } \\
\text { CTNNB1 }\end{array}$ & 225 & 89 & 5085 & 2.54 & 0.9 & 0.2 & 17.14 \\
\hline KEGG_PATHWAY & $\begin{array}{l}\text { hsa00230:Purine } \\
\text { metabolism }\end{array}$ & 14 & 0.18 & 0.02 & $\begin{array}{l}\text { POLR3K, POLA1, DCK, POLA2, HPRT1, GMPS, NME7, GART, PRIM1, } \\
\text { ADCY9, POLE2, RRM2, PKLR, RRM1 }\end{array}$ & 225 & 153 & 5085 & 2.07 & 0.91 & 0.2 & 18.08 \\
\hline KEGG_PATHWAY & $\begin{array}{l}\text { hsa03410:Base } \\
\text { excision repair }\end{array}$ & 6 & 0.08 & 0.02 & POLE2, UNG, LIG1, MBD4, NTHL1, FEN1 & 225 & 35 & 5085 & 3.87 & 0.92 & 0.19 & 18.86 \\
\hline KEGG_PATHWAY & hsa05214:Glioma & 8 & 0.1 & 0.02 & E2F1, E2F2, E2F3, CDKN2A, KRAS, CALML3, CAMK2B, PIK3R3 & 225 & 63 & 5085 & 2.87 & 0.94 & 0.2 & 21.16 \\
\hline KEGG_PATHWAY & $\begin{array}{l}\text { hsa05200: } \\
\text { Pathways in } \\
\text { cancer }\end{array}$ & 23 & 0.3 & 0.03 & $\begin{array}{l}\text { E2F1, E2F2, FZD8, CKS1B, MSH6, E2F3, HSP90AA1, PGF, FGF9, } \\
\text { SKP2, BIRC5, FZD2, CTNNB1, CTNNA2, CCNE2, CCNE1, PTK2, } \\
\text { CDKN2A, KRAS, GSK3B, PIAS2, PIK3R3, ITGA2B }\end{array}$ & 225 & 328 & 5085 & 1.58 & 0.99 & 0.27 & 30.48 \\
\hline KEGG_PATHWAY & $\begin{array}{l}\text { hsa00670:One } \\
\text { carbon pool by } \\
\text { folate }\end{array}$ & 4 & 0.05 & 0.03 & TYMS, MTHFD2, SHMT2, GART & 225 & 16 & 5085 & 5.65 & 0.99 & 0.26 & 31.03 \\
\hline KEGG_PATHWAY & $\begin{array}{l}\text { hsa04916: } \\
\text { Melanogenesis }\end{array}$ & 9 & 0.12 & 0.07 & $\begin{array}{l}\text { FZD8, KRAS, ADCY9, CALML3, GSK3B, GNAS, CAMK2B, FZD2, } \\
\text { CTNNB1 }\end{array}$ & 225 & 99 & 5085 & 2.05 & 1 & 0.47 & 57.19 \\
\hline
\end{tabular}


Table 3 KEGG pathway enrichment analysis for the genes comprising signature 3 (101.6.3) (Continued)

\begin{tabular}{|c|c|c|c|c|c|c|c|c|c|c|c|c|}
\hline KEGG_PATHWAY & $\begin{array}{l}\text { hsa05210: } \\
\text { Colorectal cancer }\end{array}$ & 8 & 0.1 & 0.08 & FZD8, MSH6, KRAS, GSK3B, BIRC5, FZD2, PIK3R3, CTNNB1 & 225 & 84 & 5085 & 2.15 & 1 & 0.48 & 60.57 \\
\hline KEGG_PATHWAY & $\begin{array}{l}\text { hsa03430: } \\
\text { Mismatch repair }\end{array}$ & 4 & 0.05 & 0.08 & RFC5, MSH6, RFC4, LIG1 & 225 & 23 & 5085 & 3.93 & 1 & 0.48 & 61.82 \\
\hline KEGG_PATHWAY & $\begin{array}{l}\text { hsa05223:Non- } \\
\text { small cell lung } \\
\text { cancer }\end{array}$ & 6 & 0.08 & 0.09 & E2F1, E2F2, E2F3, CDKN2A, KRAS, PIK3R3 & 225 & 54 & 5085 & 2.51 & 1 & 0.5 & 66.23 \\
\hline KEGG_PATHWAY & $\begin{array}{l}\text { hsa05218: } \\
\text { Melanoma }\end{array}$ & 7 & 0.09 & 0.09 & E2F1, E2F2, E2F3, CDKN2A, KRAS, FGF9, PIK3R3 & 225 & 71 & 5085 & 2.23 & 1 & 0.5 & 67.79 \\
\hline KEGG_PATHWAY & $\begin{array}{l}\text { hsa05212: } \\
\text { Pancreatic cancer }\end{array}$ & 7 & 0.09 & 0.1 & E2F1, E2F2, E2F3, CDKN2A, KRAS, PGF, PIK3R3 & 225 & 72 & 5085 & 2.2 & 1 & 0.5 & 69.77 \\
\hline
\end{tabular}

Genes comprising signature 3 (Additional file 7: Table S5) were uploaded into the DAVID gene ontology search engine (http://david.abcc.ncifcrf.gov/). KEGG pathway enrichment was generated and the table represents the output file ranked based on significance and annotated by column header. 
Table 4 KEGG pathway enrichment analysis for the genes comprising signature 3 (101.6.4)

\begin{tabular}{|c|c|c|c|c|c|c|c|c|c|c|c|c|}
\hline Category & Term & Count & $\%$ & PValue & Genes & $\begin{array}{l}\text { List } \\
\text { Total }\end{array}$ & $\begin{array}{l}\text { Pop } \\
\text { Hits }\end{array}$ & $\begin{array}{l}\text { Pop } \\
\text { Total }\end{array}$ & $\begin{array}{l}\text { Fold } \\
\text { Enrichment }\end{array}$ & Bonferroni & Benjamini & $\overline{\text { FDR }}$ \\
\hline KEGG_PATHWAY & hsa00100:Steroid biosynthesis & 3 & 0.1 & 0.03 & SQLE, FDFT1, SC4MOL & 86 & 17 & 5085 & 10.43 & 0.97 & 0.97 & 30.81 \\
\hline KEGG_PATHWAY & hsa05200:Pathways in cancer & 11 & 0.38 & 0.05 & $\begin{array}{l}\text { LAMA1, HRAS, PTK2, SOS1, CBL, VEGFA, PPARG, } \\
\text { RALA, LEF1, MDM2, LAMB1 }\end{array}$ & 86 & 328 & 5085 & 1.98 & 0.99 & 0.93 & 41.08 \\
\hline KEGG_PATHWAY & hsa04510:Focal adhesion & 8 & 0.27 & 0.05 & $\begin{array}{l}\text { LAMA1, HRAS, PTK2, FLT1, DIAPH1, SOS1, VEGFA, } \\
\text { LAMB1 }\end{array}$ & 86 & 201 & 5085 & 2.35 & 1 & 0.85 & 44.04 \\
\hline KEGG_PATHWAY & $\begin{array}{l}\text { hsa00330:Arginine and proline } \\
\text { metabolism }\end{array}$ & 4 & 0.14 & 0.06 & ARG1, P4HA2, P4HA1, CPS1 & 86 & 53 & 5085 & 4.46 & 1 & 0.82 & 49.31 \\
\hline KEGG_PATHWAY & hsa05216:Thyroid cancer & 3 & 0.1 & 0.08 & HRAS, PPARG, LEF1 & 86 & 29 & 5085 & 6.12 & 1 & 0.86 & 63 \\
\hline
\end{tabular}

Genes comprising signature 4 (Additional file 8: Table S6) were uploaded into the DAVID gene ontology search engine (http://david.abcc.ncifcr.gov/). KEGG pathway enrichment was generated and the table represents the output file ranked based on significance and annotated by column header. 


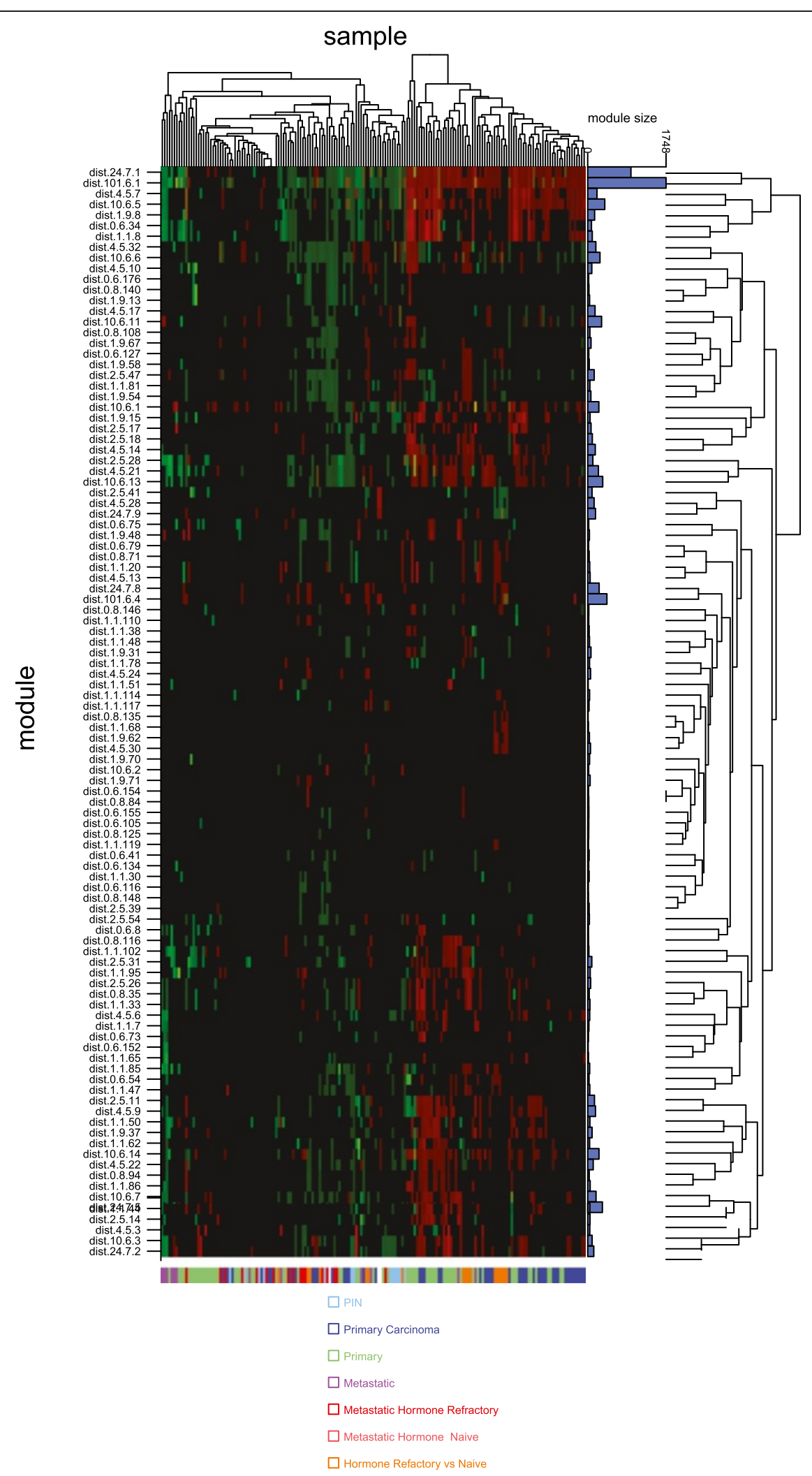

Figure 1 (See legend on next page.) 
(See figure on previous page.)

Figure 1 Gene signatures capable of discriminating between prostate cancer subgroups and classify metastatic disease. Gene signatures generated using the Varambally dataset and found to be significant discriminators of metastatic disease and primary/localised cancers (Additional file 10: Table S8) when applied to the Tomlins and Rawaswamy datasets were used to cluster samples in these datasets in a heatmap. The gene signatures represented are those capable of characterising samples from at least one progression stage (Fischer's exact $<=0.05$ ). Gene signatures are rows and samples are columns. The colour coded bar at the base of the heatmap indicates the clinical grouping for each sample as also defined in the key. Metastatic hormone refractory, metastatic hormone naïve and hormone refractory vs. naïve represent prostate cancer cases from the Tomlins dataset, as do PIN (prostatic intraepithelial neoplasia) and primary carcinoma. The other categories (metastatic and primary) are samples from the Rawaswamy dataset and are metastatic and primary cancers from multiple organ sites, not simply the prostate gland. The blue bar graph on the right-hand side of the heatmap depicts the number of genes in each signature which are differentially expressed and contribute to the sample clustering in this analysis. For signature 1 (dist 101.6 .1 and Additional file 5: Table S3) this is 1748 genes in total as highlighted and other bars are numbers of genes relative to this. The colour scale represents the mean log2 fold change for differential gene signatures ( $>=$ abs $\log 2(2))$. Red indicates module induction, green repression. Gene signatures significant in both directions are indicated in yellow. Using the mean module log2 fold change we clustered the samples and modules using hierarchical clustering with euclidean distance as a measure of dissimilarity. Data points that contained both induced and repressed values have been excluded from the clustering.

Four large gene signatures were generated at the least stringent cut-point consisting of 1334 genes referred to as signature 1 (annotated as 101.6.1: Additional file 5: Table S3), 652 genes referred to as signature 2 (annotated as 101.6.2: Additional file 6: Table S4), 836 genes referred to as signature 3 (annotated as 101.6.3: Additional file 7: Table S5) and 357 genes referred to as signature 4 (annotated as 101.6.4: Additional file 8: Table S6). Signatures 1 and 2 contained genes that predominantly discriminated between localised PCa and benign tissue. Using DAVID ontology enrichment search (http://david.abcc.ncifcrf.gov/) to determine whether KEGG pathways were enriched within these signatures, we identified focal adhesions (hsa04510: Focal adhesion, p-value $7.21 \times 10^{-6}$ ) (Table 1) as the most significant pathway for signature 1and complement and coagulation cascades for signature 2 (hsa04610: Complement and coagulation cascades, p-value 0.002) (Table 2). The 38 genes associated with the focal adhesion annotation ( $\mathrm{p}$-value $7.21 \times 10^{-6}$ ) in signature 1 are listed in Table 1 and were all significantly downregulated in metastatic samples relative to benign and localised PCa. Half of these genes were laminins (eg. laminin alpha subunit-4 (LAMA4)), integrins (eg. integrin, alpha 1 (ITGA1) and five others), thrombospondins (thrombospondins 1 and 4 (THBS1/4), collagens, actins and myosins which may reflect the remodelling of the extracellular matrix and loss of stroma in particular during the transition to metastasis. The enrichment for complement and coagulation cascades in signature 2 (p value 0.002) included complement (eg. C1R, C1QA, C3) and plasma factors as well as serpin peptidase inhibitor as listed in Table 2 and were also predominantly downregulated in metastatic cases versus benign tissue and localised PCa. Collectively, these pathway enrichments might reflect a combination of extracellular matrix changes and the contribution of infiltrating immune cells and the inflammatory response. However, given that the Varambally dataset consists of whole- tissue sections it is not possible in this meta-analysis to precisely attribute these signatures to a particular biological process.

By contrast, signatures 3 and 4 contained genes that predominantly discriminated between metastatic cases and benign tissue samples. The dominant pathway for signature 3 was cell cycle regulation (hsa04110: Cell cycle, p-value $9 \times 10^{-20}$ ) and the enrichment arose from the overexpression of a total of 36 genes linked to this process in the metastatic cases versus benign tissue. The genes are listed in Table 3 and included E2F transcription factors, DNA replication licensing factors, cyclin-dependent kinase inhibitors, cell division cycle genes and components of the mitotic spindle checkpoint control apparatus. Many of these overexpressed genes also constitute a prognostic cell cycle progression gene signature, which has been validated at the transcript level in biopsy samples [14]). For signature 4, steroid biosynthesis was the most enriched pathway (hsa00100: Steroid biosynthesis, p-value 0.03 - squalene epoxidase (SQLE), farnesyl-diphosphate farnesyltransferase 1 (FDFT1), sterol-C4-methyl oxidase-like gene (SC4MOL). In this case the enrichment was due to the differential expression of three genes that are functionally tightly linked in some cases on consecutive steps in the cholesterol biosynthesis pathway. FDFT1 was overexpressed, SC4MOL was downregulated and SQLE showed a switch in expression in which one probe on the array was repressed and another was overexpressed (Table 4). Downregulation occured predominantly in localized PCa relative to benign tissue and expression seemed higher in metastatic cases than localized prostate cancers. FDFT1 overexpression, and increases in the expression of one probe for SQLE, were most significant in the metastatic cases compared to benign tissue and localised disease. These are enzymes associated with cholesterol biosynthesis in particular and collectively catalyse 3 out of 4 consecutive reactions in the conversion of farnesyl pyrophosphate to lathosterol via squalene. FDFT1 catalyses the production of squalene from farnesyl pyrophosphate, SQLE catalyses the conversion of squalene 
Table 5 KEGG pathway enrichment for the 71-gene signature capable of subclustering localised prostate cancer cases across multiple datasets

\begin{tabular}{|c|c|c|c|c|c|c|c|c|c|c|c|c|}
\hline Category & Term & Count & $\%$ & PValue & Genes & $\begin{array}{l}\text { List } \\
\text { Total }\end{array}$ & $\begin{array}{l}\text { Pop } \\
\text { Hits }\end{array}$ & $\begin{array}{l}\text { Pop } \\
\text { Total }\end{array}$ & $\begin{array}{l}\text { Fold } \\
\text { Enrichment }\end{array}$ & Bonferroni & Benjamini & FDR \\
\hline KEGG_PATHWAY & $\begin{array}{l}\text { hsa04270:Vascular smooth muscle } \\
\text { contraction }\end{array}$ & 5 & 6.94 & 0 & $\begin{array}{l}\text { ACTG2, MYH11, KCNMB1, MYLK, } \\
\text { MYL9 }\end{array}$ & 26 & 112 & 5085 & 8.73 & 0.12 & 0.12 & 1.99 \\
\hline KEGG_PATHWAY & hsa05414:Dilated cardiomyopathy & 4 & 5.56 & 0.01 & DES, PLN, IGF1, TPM2 & 26 & 92 & 5085 & 8.5 & 0.47 & 0.27 & 9.6 \\
\hline KEGG_PATHWAY & $\begin{array}{l}\text { hsa04960:Aldosterone-regulated sodium } \\
\text { reabsorption }\end{array}$ & 3 & 4.17 & 0.02 & IGF1, ATP1A2, IRS1 & 26 & 41 & 5085 & 14.31 & 0.66 & 0.31 & 15.92 \\
\hline KEGG_PATHWAY & hsa04310:Wnt signaling pathway & 4 & 5.56 & 0.04 & $\begin{array}{l}\text { SFRP1, CAMK2G, PRICKLE2, } \\
\text { MYC }\end{array}$ & 26 & 151 & 5085 & 5.18 & 0.91 & 0.45 & 31.53 \\
\hline KEGG_PATHWAY & $\begin{array}{l}\text { hsa05410:Hypertrophic cardiomyopathy } \\
\text { (HCM) }\end{array}$ & 3 & 4.17 & 0.06 & DES, IGF1, TPM2 & 26 & 85 & 5085 & 6.9 & 0.99 & 0.57 & 49.28 \\
\hline
\end{tabular}

Genes were uploaded into the DAVID gene ontology search engine (http://david.abcc.ncifcrf.gov/). KEGG pathway enrichment was generated and the table represents the output file ranked based on significance and annotated by column header. 
Table 6 KEGG pathway enrichment analysis for the entire set of overexpressed genes in localised prostate cancer versus benign tissue in the Varambally dataset (GSE3325)

\begin{tabular}{|c|c|c|c|c|c|c|c|c|c|c|c|c|}
\hline Category & Term & Count & $\%$ & PValue & Genes & List Total & Pop Hits & Pop Total & Fold Enrichment & Bonferroni & Benjamini & FDR \\
\hline KEGG_PATHWAY & hsa00512:O-Glycan biosynthesis & 3 & 0.34 & 0.01 & $\begin{array}{l}\text { GALNTL4, GCNT1, } \\
\text { ST6GALNAC1 }\end{array}$ & 26 & 30 & 5085 & 19.56 & 0.43 & 0.43 & 8.95 \\
\hline KEGG_PATHWAY & $\begin{array}{l}\text { hsa04610:Complement and } \\
\text { coagulation cascades }\end{array}$ & 3 & 0.34 & 0.04 & C4A, C4B, SERPINA1 & 26 & 69 & 5085 & 8.5 & 0.94 & 0.75 & 36.76 \\
\hline KEGG_PATHWAY & $\begin{array}{l}\text { hsa05322:Systemic } \\
\text { lupus erythematosus }\end{array}$ & 3 & 0.34 & 0.08 & C4A, C4B, HLA-DMB & 26 & 99 & 5085 & 5.93 & 1 & 0.83 & 58.75 \\
\hline
\end{tabular}

Genes were uploaded into the DAVID gene ontology search engine (http://david.abcc.ncifcrf.gov/.). KEGG pathway enrichment was generated and the table represents the output file ranked based on significance and annotated by column header. 
to 2,3-epoxysqualene and SC4MOL catalyses the conversion of lanosterin to lathosterol. The two metabolites consecutively further downstream in the pathway are dehydrocholesterol and cholesterol. FDFT1 overexpression has previously been associated with aggressive PCa [15]). This is particularly intriguing since metastatic PCa is characterised by increases in the proliferative index of tumours [16] and the ability to produce autocrine steroid hormones from cholesterol in order to maintain androgen receptor activity [17]. Consequently the observation of increased levels of these enzymes in metastatic cases may hypothetically imply enhanced cholesterol biosynthesis to sustain its use for steroid hormone biogenesis by the tumours.

Discrimination between cancer and benign control tissue and also between metastatic disease and other clinical cases represents an important goal of biomarker research. Thus, we used these gene signatures to classify clinical samples in prostate cancer samples and metastatic tissue samples in two additional datasets. One consisted of prostate cancer samples isolated by laser

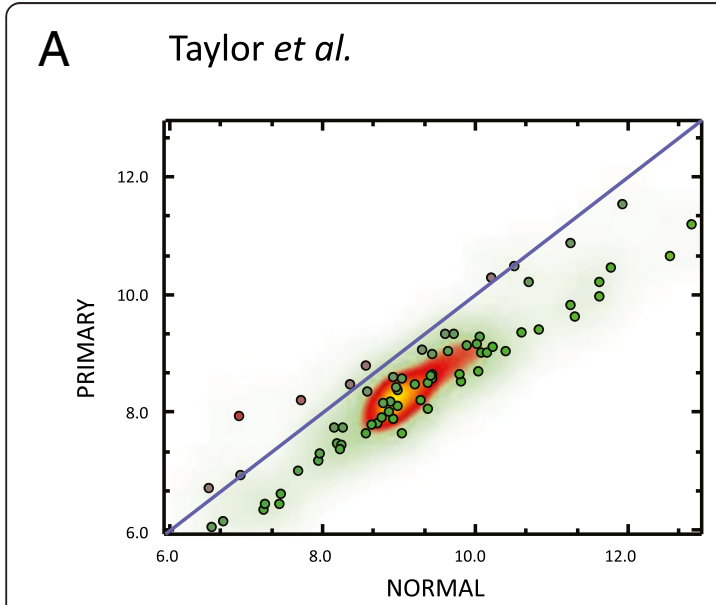

SYMBOL NORMAL PRIMARY FOLD CHANGE

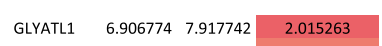

\begin{tabular}{|l|l|l|l|}
\hline GLATL1 & 6.906774 & 7.917742 & 2.015263 \\
\hline MYC & 7.707574 & 8.185126 & 1392379 \\
\hline
\end{tabular}

\begin{tabular}{ll|rr|r|}
\hline MYC & 7.707574 & 8.185126 & 1.392379 \\
\hline FMOD & 8.560921 & 8.76869 & 1.154901 \\
\hline
\end{tabular}

\begin{tabular}{|l|r|r|r|}
\hline FMOD & 8.560921 & 8.76869 & 1.154901 \\
\hline CCL4 & 6.514742 & 6.676367 & 1.118547 \\
\hline
\end{tabular}

\begin{tabular}{l|l|l|l|}
\hline ZNF615 & 8.361406 & 8.461288 & 1.071686 \\
\hline
\end{tabular}

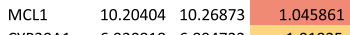

\begin{tabular}{|l|l|l|l|}
\hline CYP39A1 & 6.920818 & 6.894722 & -1.01825 \\
\hline FOS & 10.50548 & 10.46167 & -1.03083 \\
\hline
\end{tabular}

\begin{tabular}{|l|l|l|l|}
\hline FOS & 10.50548 & 10.46167 & -1.03083 \\
\hline
\end{tabular}

\begin{tabular}{|l|l|l|l|}
\hline EBPL & 8.575874 & 8.323491 & -1.19117 \\
\hline
\end{tabular}

\begin{tabular}{llll|r} 
FAM36A & 9.301839 & 9.045351 & -1.19457 \\
\hline CYR61 & 9.596716 & 9.309407 & -1.22036
\end{tabular}

\begin{tabular}{llll|r}
\hline ADARB1 & 8.918336 & 8.577855 & -1.26618 \\
\hline
\end{tabular}

\begin{tabular}{llll|l|}
\hline ADARB1 & 8.918336 & 8.577855 & -1.26618 \\
\hline C3orf64 & 11.23722 & 10.86437 & -1.29491 \\
\hline
\end{tabular}

\begin{tabular}{|l|l|l|l|}
\hline C3orf64 & 11.23722 & 10.86437 & -1.29491 \\
\hline IGF1 & 11.92109 & 11.51532 & -1.3248 \\
\hline
\end{tabular}

\begin{tabular}{lllll} 
DPP4 & 9.722254 & 9.315641 & -1.32557 \\
\hline
\end{tabular}

\begin{tabular}{llll|l|}
\hline FOXF1 & 8.153576 & 7.719392 & -1.35115 \\
\hline
\end{tabular}

\begin{tabular}{lllll} 
SIN3A & 9.432331 & 8.954617 & -1.39254 \\
\hline
\end{tabular}

\begin{tabular}{lllll} 
IRS1 & 9.029387 & 8.544991 & -1.399 \\
\hline
\end{tabular}

\begin{tabular}{lllll} 
MXRA7 & 10.68732 & 10.19753 & -1.40424 \\
\hline
\end{tabular}

\begin{tabular}{llll|l|}
\hline PAGE4 & 6.544602 & 6.022728 & -1.43582 \\
\hline
\end{tabular}

\begin{tabular}{|l|l|l|r|}
\hline GBP1 & 8.261975 & 7.721639 & -1.45431 \\
\hline SFRP1 & 8.965537 & 8395006 & -1.48507 \\
\hline
\end{tabular}

\begin{tabular}{lllll|l} 
SFRP1 & 8.965537 & 8.395006 & -1.48507 \\
\hline PIN & 6.697379 & 6.117262 & -1.4997
\end{tabular}

\begin{tabular}{lllll}
8.965537 & 8395006 & -1.48507 \\
6.697379 & 6.117262 & -1.49497 \\
\hline
\end{tabular}

$\begin{array}{lllll}\text { SLC22A3 } & 9.639426 & 9.015032 & -1.54156\end{array}$

\begin{tabular}{ll|l|l|l|}
\hline OSR2 & 8.790582 & 8.122536 & -1.58892 \\
\hline
\end{tabular}

\begin{tabular}{llll|l} 
CLIC6 & 7.684455 & 6.989104 & -1.61928 \\
\hline
\end{tabular}

\begin{tabular}{llll} 
PABPC4L & 7.962172 & 7.264653 & -1.6217 \\
\hline
\end{tabular}

\begin{tabular}{|l|l|l|l|}
\hline MRGPRF & 8.87798 & 8.158198 & -1.64693 \\
\hline
\end{tabular}

\begin{tabular}{l|lll|l}
\hline CLIP4 & 8.191268 & 7.455136 & -1.6657 \\
\hline
\end{tabular}

\begin{tabular}{llll} 
PDE4D & 9.214379 & 8.457332 & -1.69003 \\
FHI2 & 9.8855 & 9.111159 & -1.71041 \\
\hline
\end{tabular}

\begin{tabular}{lrrrr} 
PHL2 & 9.8855 & 9.111159 & -1.7104 \\
\hline LTF & 10.05994 & 9.272211 & -1.72635
\end{tabular}

\begin{tabular}{llll|l|} 
& 10.05994 & 9.272211 & -1.72635 \\
\hline
\end{tabular}

\begin{tabular}{llrr|r}
\hline OGN & 7.950126 & 7.146403 & -1.7456 \\
\hline
\end{tabular}

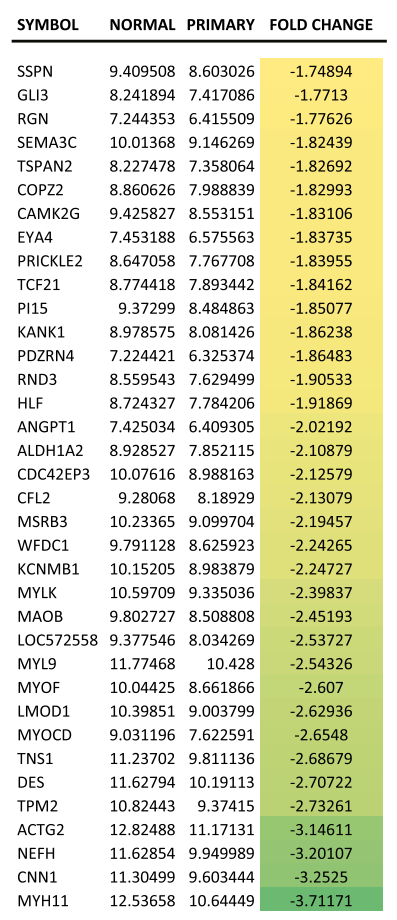

B TCGA-PRAD

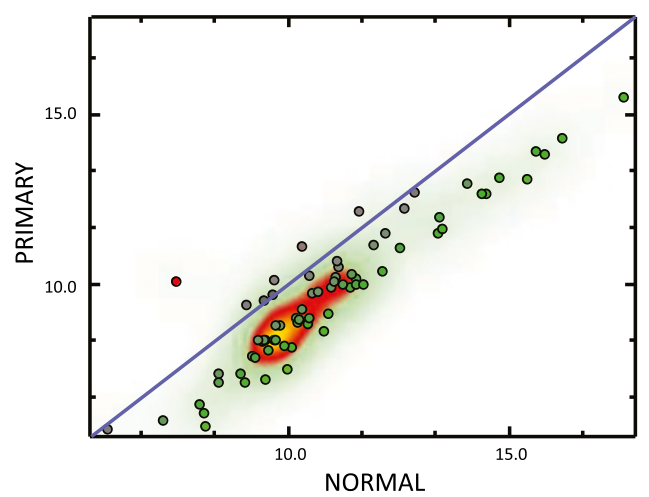

\begin{tabular}{|c|c|c|c|}
\hline MBOL & NORMAL & PRIMARY & CHANC \\
\hline CLIP4 & 9.67412 & 8.298259 & -2.59523 \\
\hline CDC42EP3 & 11.52193 & 10.13509 & -2.61505 \\
\hline OGN & 10.2151 & 8.825615 & -2.61985 \\
\hline HLF & 9.724102 & 8.311322 & -2.6625 \\
\hline TSPAN2 & 9.258912 & 7.781335 & -2.78481 \\
\hline NEFH & 13.43626 & 11.93944 & -2.8222 \\
\hline WFDC1 & 10.46986 & 8.946525 & -2.87454 \\
\hline MXRA7 & 12.54074 & 11.00498 & -2.89942 \\
\hline CFL2 & 11.39027 & 9.840245 & -2.92821 \\
\hline PLN & 9.562167 & 8.00411 & -2.94457 \\
\hline МАOB & 11.52369 & 9.95599 & -2.96432 \\
\hline CLIC6 & 8.907655 & 7.336251 & -2.97194 \\
\hline RGN & 8.004386 & 6.411789 & -3.01592 \\
\hline RND3 & 10.43499 & 8.778803 & -3.15182 \\
\hline TPM2 & 14.7658 & 13.0901 & -3.19475 \\
\hline MYL9 & 15.60606 & 13.84934 & -3.37929 \\
\hline PRICKLE2 & 9.897849 & 8.139418 & -3.3833 \\
\hline MSRB3 & 11.69072 & 9.928429 & -3.39237 \\
\hline TNS1 & 14.38974 & 12.61048 & -3.4325 \\
\hline MYOF & 12.12388 & 10.34144 & -3.44006 \\
\hline KCNMB1 & 10.9181 & 9.0827 & -3.56869 \\
\hline CNN1 & 14.48048 & 12.61957 & -3.63236 \\
\hline LTF & 13.49969 & 11.59508 & -3.74407 \\
\hline EYA4 & 8.077518 & 6.155506 & -3.78951 \\
\hline DES & 16.19062 & 14.25205 & -3.83326 \\
\hline LMOD1 & 13.3857 & 11.44545 & -3.8377 \\
\hline PDZRN4 & 9.014486 & 7.061059 & -3.87293 \\
\hline ALDH1A2 & 10.06106 & 8.094925 & -3.90721 \\
\hline ACTG2 & 15.82486 & 13.78335 & -4.11676 \\
\hline MYH11 & 17.61345 & 15.45035 & -4.47875 \\
\hline ATP1A2 & 10.79166 & 8.578395 & -4.63724 \\
\hline MYOCD & 9.47278 & 7.125689 & -5.08797 \\
\hline MYLK & 15.42531 & 13.05382 & -5.17478 \\
\hline LOC572558 & 8.136008 & 5.759487 & -5.19283 \\
\hline ANGPT1 & 9.969429 & 7.461877 & -5.68654 \\
\hline
\end{tabular}

Figure 2 Differential expression of a 71-gene signature classifier in a prostate cancer exon-array dataset (Taylor et al.) and the TCGA RNA-seq dataset for prostate cancer (TCGA-PRAD). The expression values of the 71-gene signature (dist.0.6.34) capable of subclustering localised prostate cancer from other samples in all three interrogated datasets are shown in two independent datasets, $\mathbf{A}$. a prostate cancer exon-array dataset (Taylor et al.) and B. TCGA RNA-seq dataset for prostate cancer (TCGA-PRAD) were used. Values were log2 normalized and the mean of the sample groups (PRIMARY TUMOUR/SOLID TISSUE NORMAL) is shown. 
Table 7 Comparison of the performance of a 31-gene signature with ERG, AR and KLK3 in discriminating between benign tissue, localised prostate cancer and metastatic disease

\begin{tabular}{llll}
\hline Gene/signature & AUC benign-local & AUC benign-metastatic & AUC localized-metastatic \\
\hline KLK3 & 0.5204082 & 0.9104938 & 0.8707483 \\
ERG & 0.812616 & 0.9326599 & 0.6099773 \\
AR & 0.6581633 & 0.8395062 & 0.8435374 \\
31 Gene signature & 0.994898 & 0.9938272 & 0.957672 \\
Derived from the Grasso Data & & & \\
\hline
\end{tabular}

Data were downloaded from Grasso et al.,. ROC statistics were computed in an evaluation sample set having established the weighting for genes in the signature using logistic regression in a test sample set. We report the area under the curve (AUC) for each transcript and for the signature for each of three pairwise comparisons as generated using the R package ROCR.

capture microdissection generated by Tomlins et al. [5] and the other contained expression array data from primary and metastatic tumours from multiple tissue sites generated by Ramaswamy et al. [6].

The Tomlins dataset consisted of various refined subgroups based on isolation of cell sub-populations including stromal fractions, epithelial fractions, localised prostate cancer and hormone-naïve and refractory metastatic disease. The Ramaswamy et al. dataset consisted of cancers from 14 organ sites with paired normal samples as well as normal tissues. In each dataset, we asked whether our signatures and sub-signatures could discriminate between the sample groups. To determine this, we first assessed the mean foldchange in the expression of each gene signature in each sample group in both datasets (Additional file 9: Table S7). We then performed a Fischer's Exact test to identify signatures that were capable of discriminating between localised prostate cancer, metastatic prostate cancer and the other sample groups defined in all three published studies Varambally et al., Tomlins et al., and Ramaswamy et al. (refer to Materials and Methods for more detail on subgroups/sample types) (Additional file 10: Table S8). Gene ontologies were assigned to these statistically significant gene clusters and the clustering is represented in a heatmap for the classifying modules combining both the Tomlins and Ramaswamy sample sets (Figure 1 and Additional files 10 and 11: Tables S8 and S9 for gene ontology annotations). The smallest gene signature (dist.0.6.34) capable of subclustering localised prostate cancer from other samples in all three datasets consisted of 71 genes (Additional file 12). This small signature was a sub-component of the original signature 1 (101.6.1). The most significantly enriched biological process associated with these genes was vascular smooth muscle contraction (hsa04270: Vascular smooth muscle contraction, p-value $2 \times 10^{-3}$ ) (Table 5). The four genes within this signature that were individually most significantly overexpressed in localised prostate cancers compared to benign tissues and metastatic cases were an oncogenic transcription factor, v-myc avian myelocytomatosis viral oncogene homolog (MYC), a proteoglycan capable of sequestering transforming growth factor beta called fibromodulin (FMOD), a mitochondrial enzyme associated with fatty acid metabolism called glycine $\mathrm{N}$-acyltransferaselike protein 1 (GLYATL1) and an extraneuronal monoamine transporter called solute carrier family 22 member 3 (SLC22A3). MYC has been shown to be overexpressed in prostate cancer [18] and to drive tumourigenesis in a transgenic model of the disease [19]. Fibromodulin has not been widely studied in cancer and has not been implicated in prostate cancer. It is, however, known to be significantly overexpressed in chronic lymphocytic leukemia (CLL) versus normal B lymphocytes [20] and associated with a resistance signature to DNA damage-induced apoptosis in CLL [21]. Furthermore, the expression of fibromodulin is known to be induced in leiomyoma in response to TGF-beta through Smad and MAP kinase signalling [22]. GLYATL1 has not been associated with cancers. SLC22A3 has been reported to be overexpressed in localised prostate cancer at the transcript level when compared to benign tissue [5].

The other genes within this coexpression signature were downregulated in prostate cancers versus benign tissue and the majority were myosins, such as myosin, heavy polypeptide 11, smooth muscle (MYH11), myocardin (MYOCD), and myosin, light chain 9, regulatory (MYL9)thus accounting for the pathway enrichment for vascular smooth muscle contraction. As prostate cancer progresses to more advanced stages there is a depletion of stromal cells from the tissue and this perhaps explains the dominant contribution from downregulated muscleassociated genes to the signature and also other features of pathway enrichments particularly of the focal adhesion classification [23]. In order to determine whether our signature was consistent across more recent datasets, we downloaded an exon-array dataset generated by Taylor et al., and also The Cancer Genome Atlas (TCGA) data recently generated using high-throughput transcript sequencing of prostate cancers [24] (data generated by the data generated by the TCGA Research Network: http:// cancergenome.nih.gov/). MYC and GLYATL1 remain significantly overexpressed features ( $>1.3$ fold) within these signatures in both datasets (Figure 2) with the vast majority of other gene transcripts downregulated including those enriched in the KEGG pathway analysis for vascular smooth muscle contraction. 
Whilst our 71 gene signature mainly contains differentially expressed genes that are downregulated in cancers versus benign tissues, most prostate cancer biomarkers that are currently under evaluation are overexpressed transcripts and proteins in the disease state. Consequently, we next sought to evaluate genes that were overexpressed in localised prostate cancers in signatures 1-4 more thoroughly in other datasets. There were 97 annotated gene transcripts in total overexpressed (Additional file 13). We had previously performed pathway analyses on signatures 1-4 which included both up- and downregulated genes (Tables 1, 2, 3 and 4). We now repeated this solely for the 97 overexpressed genes and this yielded pathway enrichment for O-glycan biosynthesis (hsa00512: O-glycan biosynthesis, p-value 0.009) as the most significant KEGG enrichment (Table 6).

To further refine this gene set, we then interrogated the Oncomine compendium of expression array data to determine which of these 97 genes are significantly overexpressed in at least three additional independent prostate cancer datasets when a Top 1\% overexpression threshold was applied together with a p-value threshold of $1 \times 10^{-4}$ [25]. Thirty three annotated genes, around one-third of the 97-gene set fulfilled these criteria. This included 3 of the 4 overexpressed genes (MYC, GLYATL1 and SLC22A3) in the 71 gene signature subgrouping prostate cancers in the Varambally, Tomlins and Ramaswamy studies (highlighted in bold in Additional file 13). This 33 gene set also included four of the five glycosylating enzymes (UDP N-acetylglucosamine pyrophosphorylase 1 (UAP1), glucosaminyl (N-acetyl) transferase 1 , core 2 (GCNT1), beta-1,3-glucuronyltransferase 1 (B3GAT1) and RAP1 GTPase activating protein 2 (RAP1GAP2/ GARNL4)) contributing to the ontology enrichment for glycan biosynthesis in the larger 97 gene set. Notably, others and we have recently reported that UAP1 and GCNT1 are overexpressed in prostate cancer tissue using immunohistochemistry. In addition, an aminosugar conjugate, O-linked $\mathrm{N}$-acetylglucosamine (O-GlcNAc), is also significantly elevated in prostate cancer [26,27]. Furthermore, the UAP1 transcript has also been reported to be detectable in urine and plasma samples as a component of a multi-gene signature [28]. Additionally, UDP sugar conjugates have been identified as elevated in prostate cancers through metabolomics and O-linked $\mathrm{N}$-acetylglucosamine is an overexpressed prostate cancer tissue biomarker, which can be conjugated to a variety of proteins to affect their stability and activity including c-Myc [29,30]. Consequently, the presence of these genes encoding glycosylating enzymes within this signature has been partly validated in tissue at the proteins level and suggests that more systematic profiling of glycoproteins may reveal new biomarkers.

Biologically, it is interesting to consider what might contribute to the increased expression of these genes in prostate cancers. Prostate cancer is driven by the dysregulated expression and activity of a number of transcription factors. The most notable example is the androgen receptor but others are overexpressed through chromosomal rearrangements and gene fusions as well as copy number variation as prostate cancer develops and progresses. This in turn has a significant impact on the expression of gene targets for these transcription factors and makes it plausible that a proportion of overexpressed genes reflect changes in transcription factor expression and activity. In this context, it is noteworthy that a total of five transcription factors were present in this group of 33 annotated genes ((single-minded family bHLH transcription factor 2 (SIM2), MYC, distal-less homeobox 1 (DLX1), homeobox C6 (HOXC6) and v-ets avian erythroblastosis virus E26 oncogene homolog (ERG)).

c-Myc is a well-established oncogenic transcription factor, which is overexpressed through chromosomal amplification on $8 \mathrm{q} 24$ but also through post-translational events, which may include glycosylation of the $\mathrm{N}$-terminal transactivation and concomitant antagonism of proteasomal degradation [18,31,32]. ERG is part of a highly prevalent gene fusion affecting chromosome 21 and driven by the activity of the AR [33]. It is overexpressed in around 50\% of prostate cancers through a chromosomal rearrangement, which fuses it to the upstream androgen receptordependent regulatory element controlling TMPRSS2 expression. SIM2 overexpression is associated with changes in transcriptional control affecting other loci on chromosome 21 [34,35]. Target genes for MYC and ERG have been extensively explored in clinical and cell-line datasets using expression array profiling with targeted knockdown and overexpression in prostate cancer cells. These approaches have linked MYC to processes including ribosome biogenesis and splicing and ERG to cell motility and migration, respectively [36-38]. Whilst the 33 genes did not include significant number of established MYC target genes, several reported ERG target were present including B3GAT1, phospholipase A1 member A (PLA1A) and collagen, type IX, alpha 2 (COL9A2) [39]. In addition, there were a number of direct AR targets including UAP1 and GCNT $[30,40]$. Importantly, whilst the AR is the principal transcription factor driving all stages of prostate cancer development, its target genes cannot be easily inferred by coexpression with the AR in contrast to ERG relative to ERG target genes. Target genes for HOXC6, SIM2 and DLX1 are less well defined in prostate cancers but given the presence of ERG and AR target genes within this geneset it is highly likely that they also contribute, being transcription factors, to the expression of some of these genes. A more systematic understanding of the interplay between these transcription factors and dependent gene networks will emerge in future studies. This will require targeting the expression of the transcription factors in experimental model systems and profiling concomitant changes in 
transcription factor recruitment, chromatin architecture and gene expression.

In the interim, however, it was possible to infer codependency based on co-clustering of genes in clinical samples. We did so in two additional datasets, an exon-array dataset generated by Taylor et al. and a transcriptomic dataset generated for prostate cancer through highthroughput sequencing by the The Cancer Genome Atlas (TCGA) (Figure 3). In both datasets, we were able to firstly reconfirm the ability of these 33 genes to discriminate between localised prostate cancer and benign tissue samples (Figure 3). Secondly, ERG co-clustered within these 33 genes with bona fide target genes such as B3GAT1 and PLA1A corroborating a contribution at least from ERG to this prostate cancer-specific overexpression signature [39]. Intriguingly, another transcription factor, DLX1, also coclustered with ERG raising the possibility of a transcription factor hierarchy in which early emergence of an ERG gene fusion may trigger aberrant expression of other developmental transcription factors.

Currently prostate-specific antigen (PSA)/kallikrein 3 (KLK3) is the most widely used protein biomarker for prostate cancer. The androgen receptor (AR) is the most significant transcription factor driving prostate cancer, but is also expressed at high levels in untransformed luminal epithelial cells and therefore is predominantly used as a transcript biomarker associated with metastatic disease and concomitant copy-number amplification [41]. Gene fusions have been detected which significantly elevate the transcript levels of ETS transcription factors and the most prevalent example in prostate cancer is the TMPRSS2-ERG fusion [33]. Detection of the fusion has been reported in biological fluids including urine samples [42].

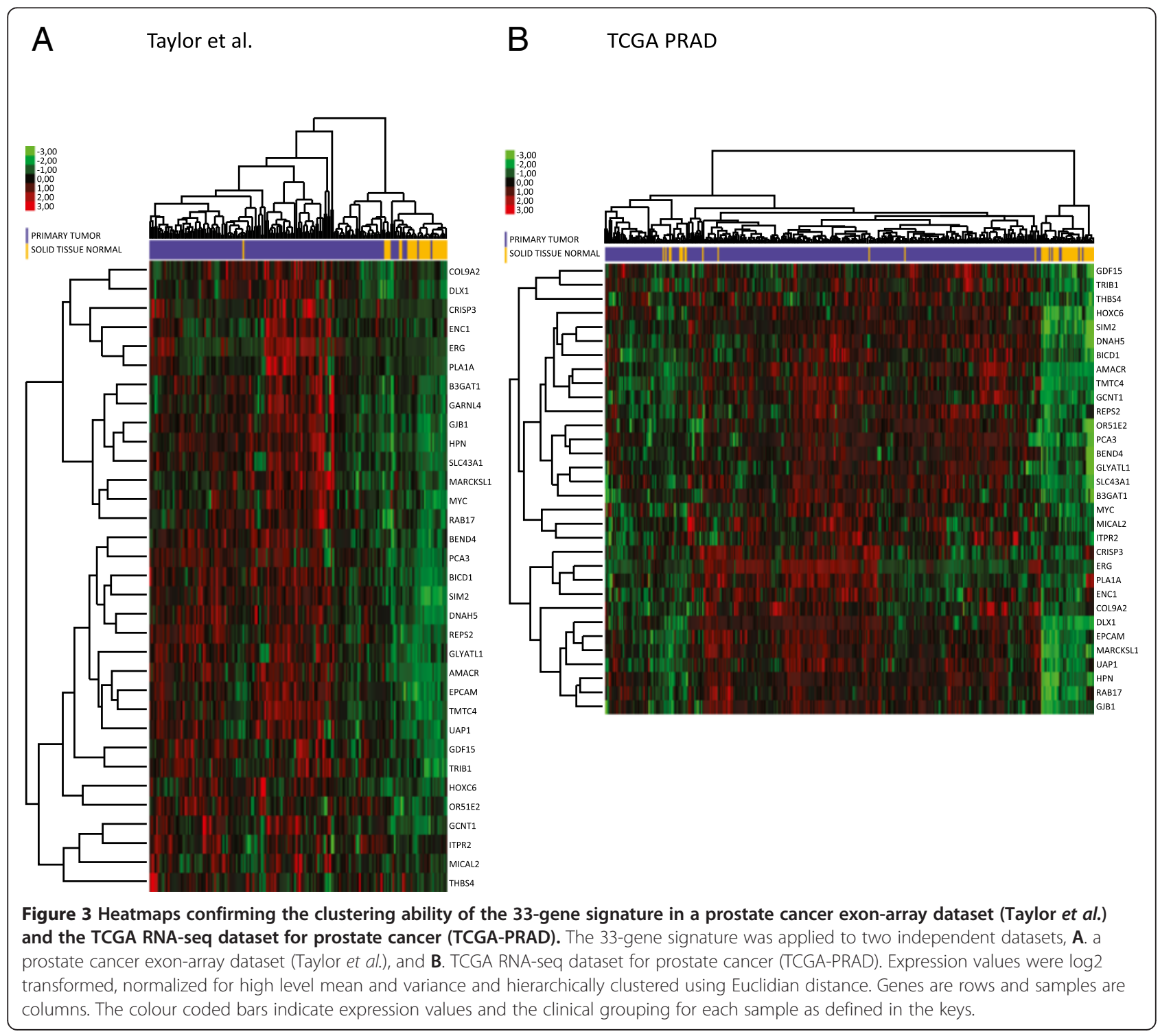




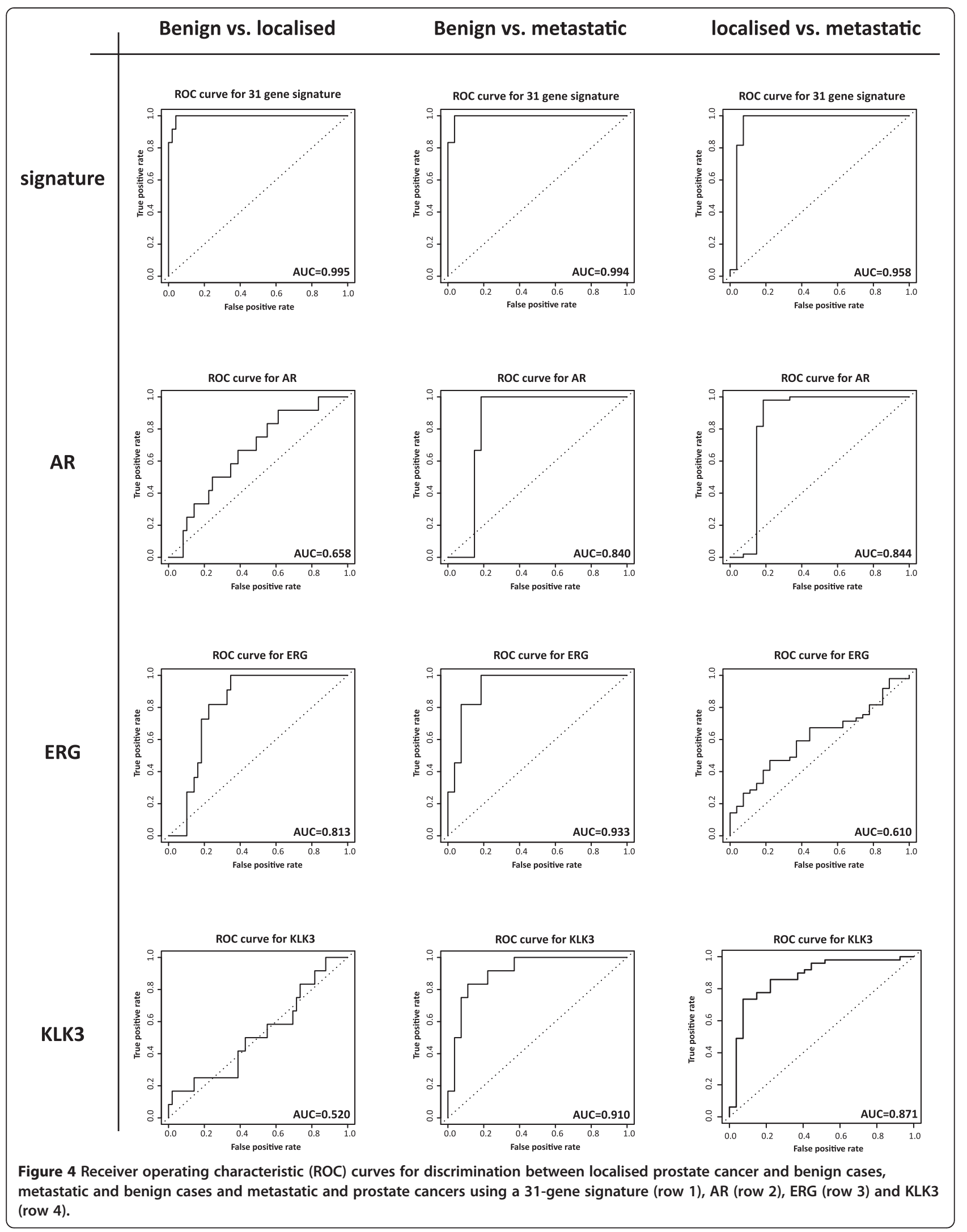




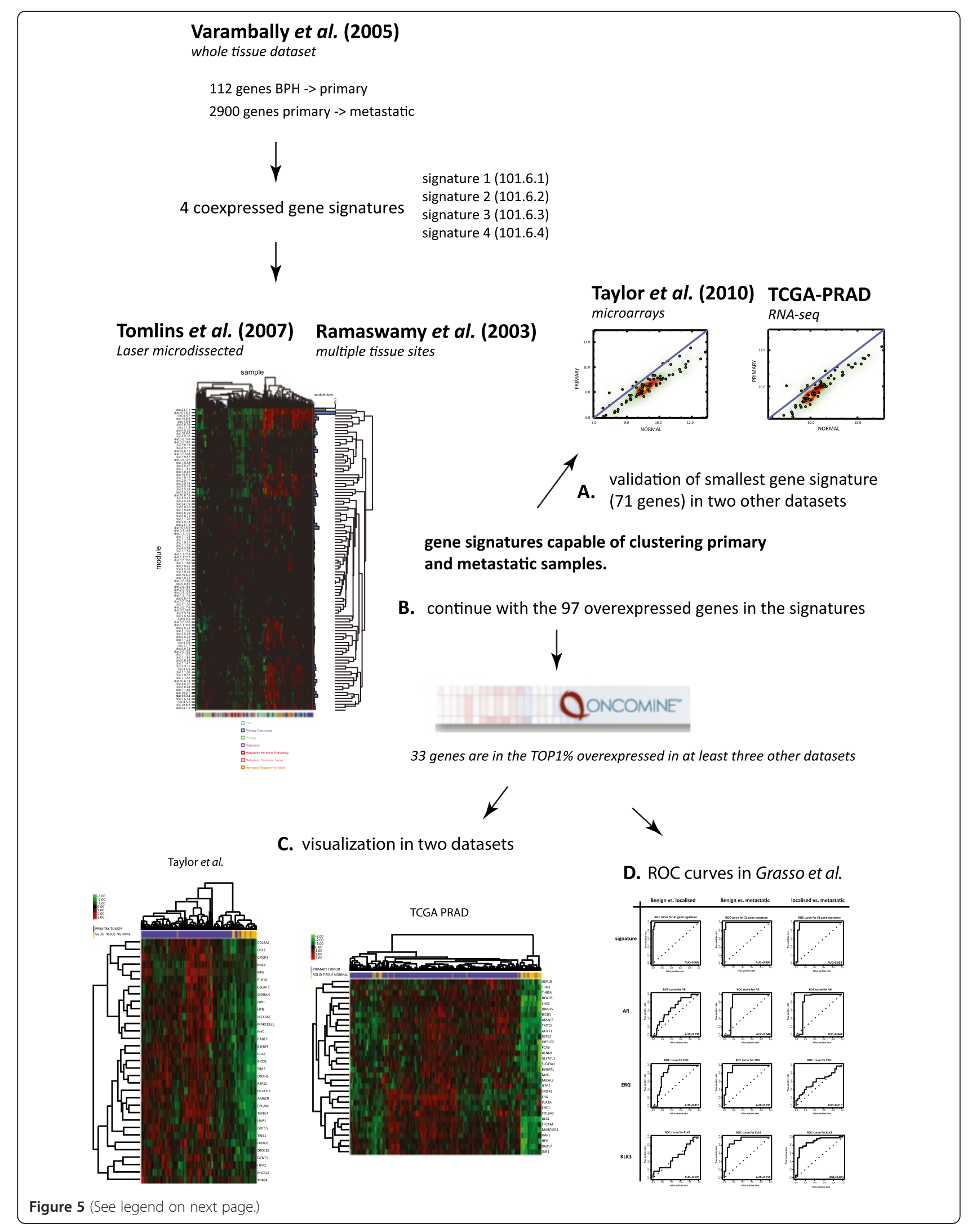




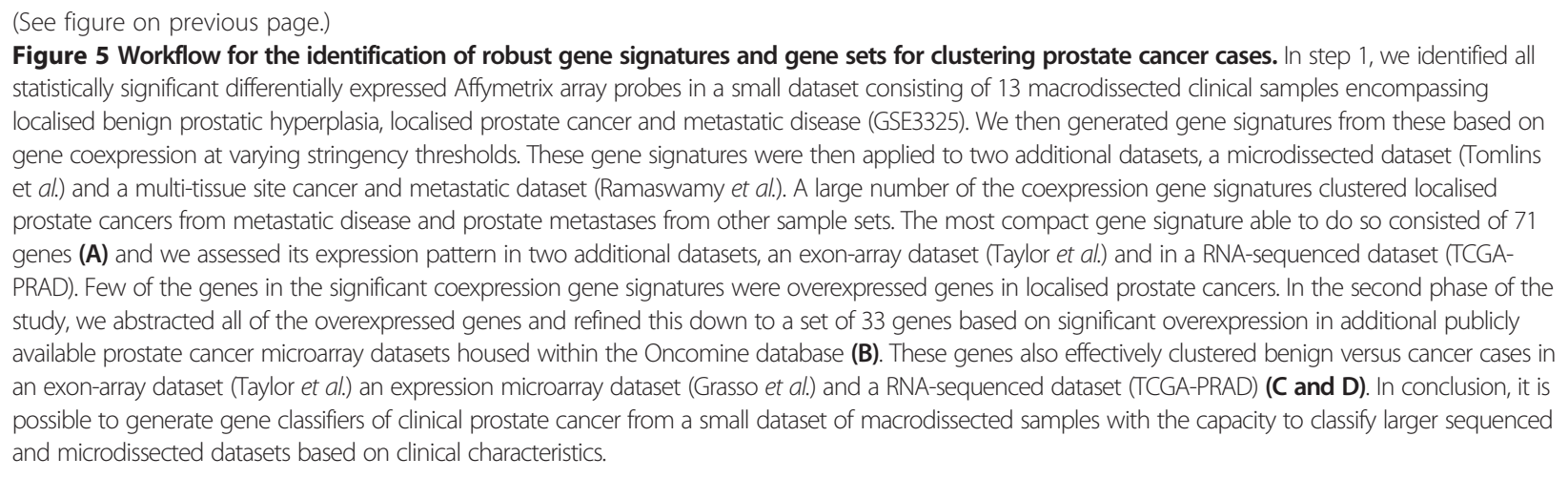

To assess the performance of the 33-gene signature in comparison to KLK3/PSA, AR and ERG we interrogated an additional independent expression array dataset generated by Grasso et al., and consisting of benign tissue, localised prostate cancer and metastatic cases [43]. This dataset was generated using two different array platforms on distinct sets of samples (Methods section). Cysteine-rich secretory protein-3 (CRISP3) was excluded from the signature due to missing values in the datasets for this gene and prostate cancer antigen 3 (PCA3) was not represented on the arrays leaving a 31-gene signature for evaluation. In the first phase of the signature evaluation we assessed the weighted contribution of each gene in the signature using a logistical regression model on a training dataset consisting of the samples profiled on an Agilent oligo microarray platform. We then used the samples profiled on a second platform, the $4 \times 44 \mathrm{~K}$ Agilent microarray to evaluate the performance of the signature and compared this to KLK3, ERG and AR. Three pairwise sample comparisons were undertaken benign versus localised prostate cancer, benign versus metastatic cases and localised prostate cancers versus metastatic cases. Whilst all three transcripts and the signature discriminated between metastatic samples and benign tissue with good specificity and sensitivity as reflected in an area-under-the-curve (AUC) ranging from 0.83 for the AR to 0.99 for the signature, only the signature provided an AUC of $\geq 0.95$ for all three pairwise comparisons (Table 7). Since both the AR and KLK3 are expressed in both untransformed prostate cells and prostate cancer it is perhaps not surprising that neither yielded an AUC of $>0.65$ in discriminating between localised prostate cancer and benign tissue samples. By contrast ERG expression is driven by a cancer-associated gene fusion and the AUC was 0.81 (Table 7). AR is amplified and overexpressed in metastatic prostate cancers and this likely explains the higher AUC for this marker (0.84) in discriminating metastatic cases from localised prostate cancers [41]. KLK3/PSA was also higher, 0.87, in this context. ERG by contrast whilst consistently overexpressed in the majority of localised prostate cancers is of variable utility as a prognostic marker according to the study cohort examined associating variously positively or negatively with progression and metastasis [44-46]. In our evaluation the AUC for ERG in discriminating localised prostate cancer from metastatic cases was 0.61 , performing more poorly than as a discriminator of localised prostate cancer from benign tissue samples. The AUC differences between the markers and the signature in each pairwise comparison of the sample sets was also visualised in receiver operating characteristic (ROC) curves (Figure 4). These comparisons highlight the importance of using a multi-gene signature since no single gene provides robust discrimination at all stages of the disease, no doubt reflecting changes in the underlying biological drivers during disease progression. We provide in addition AUC values for each individual gene and array probe for each of the pairwise sample comparisons in the test set (Additional file 14: Table S12) and the validation set (Additional file 15: Table S13). Although beyond the scope of this paper we hope that this will assist in further evaluation of the signature by researchers in the field.

\section{Conclusions}

In conclusion, in this study we have used a multi-step approach to refine gene signatures derived from diverse transcript detection platforms and sample types in order to arrive at a robust gene signature able to discriminate between PCa and benign tissue (Figure 5). This is the first time that this has been attempted and demonstrates that value exists in transcript signatures generated from amongst the earliest microarray studies right through to high-throughput sequencing. In brief, beginning with a small expression array dataset consisting of 13 macrodissected samples, we have been able to derive gene signatures capable of subclustering localised PCa and metastases in a larger microdissected and a multi-cancer dataset (Figure 5). This highlights that there are valuable gene transcript signatures that can be robust despite cellular heterogeneity in 
PCa and the evolution of transcript detection technologies. In addition, we have discovered that gene transcripts that are significantly overexpressed within these signatures are also overexpressed in much more recently acquired exonarray and sequence-based TCGA data, transcript detection platforms that were unavailable when the Varambally, Tomlins and Ramaswamy studies were undertaken (Figure 5). Finally, we have evaluated the performance of these transcripts as a signature in discriminating between benign tissue samples, localised PCa and metastatic disease in an additional dataset generated by Grasso et al. ROC curves reveal that the signature exceeds the performance of ERG, KLK3 or the AR as a classifier. Intriguingly, one third of these genes are glycosylating enzymes and transcription factors. PCa is significantly driven by a transcription factor, the AR, but there is increasing evidence of contributions by others and of interplays between them and indeed our signature does not include the AR itself. However, it includes both established examples (MYC and ERG) but also others that have so far been less studied (SIM2, DLX1 and HOXC6). Mechanistically, future work will investigate this transcriptional co-dependency in more detail and clinically these signatures will be further evaluated in clinical cohorts.

\section{Additional files}

Additional file 1: Figure S1. W(C) values generated from branch length thresholds plotted against the number of clusters produced for single, average, complete, ward and mcquitty agglomerative hierarchical and divisive clustering methods.

Additional file 2: Table S1. Annotated Affymterix probes for differentially expressed genes in localised prostate cancer and metastatic tumours. Genes differentially expressed within dataset GSE3325. Columns 1 and 2 represent Affymetrix probe identifiers and gene symbols, respectively. Columns 3-5 represent mean probe signals for benign, prostate cancer and metastatic samples. Columns 6-9 represent a linear fold change and corresponding $p$-value for each contrast. Genes are ranked based on overexpression in localised prostate cancer versus benign tissue. Differential expression and associated statistical significance ( $p$-values) have been calculated as described in the methods/supplementary methods section

Additional file 3: Figure S2. $H(X) G O$ bit values generated from branch length thresholds plotted against the number of clusters produced for single, average, complete, Ward and Mcquitty agglomerative hierarchical and divisive clustering methods.

Additional file 4: Table S2. Differentially expressed genes within Varambally dataset (accession number GSE3325) grouped into coexpression signatures using the Ward agglomerative method. Genes are ranked based on overexpression in localised prostate cancer versus benign tissue. Annotation includes the cytogenetic locus for each gene and the gene name. Coexpression modules are defined at multiple branch thresholds. Nine branch thresholds were used. Dist.0.6 represents the most compact modules at the highest stringency threshold and dist.101.6 representing the least compact modules at the lowest stringency threshold. Numbers attributed to each gene within each branch threshold define membership of a distinct coexpression module. With reference to the main text 'signature 1' corresponds to 101.6.1, 'signature 2' corresponds to 101.6.2, 'signature 3' corresponds to 101.6.3 and 'signature 4' corresponds to 101.6.4.
Additional file 5: Table S3. Differentially expressed genes comprising signature 1. Genes within signature 1 and sub-signatures defined at more stringent branch thresholds are ranked based on overexpression in localised prostate cancer within dataset GSE3325. Annotation includes the cytogenetic loci, gene names and unigene modules.

Additional file 6: Table S4. Differentially expressed genes comprising signature 2. Genes within signature 2 and sub-signatures defined at more stringent branch thresholds are ranked based on overexpression in localised prostate cancer within dataset GSE3325. Annotation includes the cytogenetic loci, gene names and unigene modules.

Additional file 7: Table S5. Differentially expressed genes comprising signature 3. Genes within signature 3 and sub-signatures defined at more stringent branch thresholds are ranked based on overexpression in localised prostate cancer within dataset GSE3325. Annotation includes the cytogenetic loci, gene names and unigene modules.

Additional file 8: Table S6. Differentially expressed genes comprising signature 4. Genes within signature 4 and sub-signatures defined at more stringent branch thresholds are ranked based on overexpression in localised prostate cancer within dataset GSE3325. Annotation includes the cytogenetic loci, gene names and unigene modules.

Additional file 9: Table S7. Fold-change in coexpressed gene signatures derived from Varambally et al., in samples from a laser capture microdissected prostate cancer dataset and a multi-cancer dataset. All signatures and sub-signatures generated from Varambally et al. were tested for enrichment in individual samples from two additional datasets

- a laser-capture microdissected prostate cancer dataset generated by Tomlins et al., and a multi-cancer dataset generated by Ramaswamy et al., Column A and B are collectively signature identifiers indicating the threshold for coexpression (column A) and the signature/module number (column B). Column C indicates the overall direction of the fold-change indicating overexpression ('induced') or downregulation ('repressed'). Only signatures achieving a fold-change of at least two-fold are listed. Columns C through to CX represent individual samples from the Tomlins study. The data are listed sample-by-sample in order of clinical progression beginning with prostatic intraepithelial neoplasia ('PIN') cases and progressing to localised prostate cancer ('Primary Carcinoma'), followed by metastatic hormone naive cases and finally metastatic hormone refractory samples. The GSM accession code provided for each sample is traceable through the NCBI GEO data repository from which the data were downloaded. Finally there are columns representing pairwise sample comparisons between metastatic hormone refractory and naiive samples. Fold-change information for the data for samples from Ramaswamy et al. is represented in column CY onwards.

Additional file 10: Table S8. Significance values and overall direction of the differential expression of gene signatures derived from Varambally et al., in samples from a laser capture microdissected prostate cancer dataset and a multi-cancer dataset. All gene signatures are classified with aggregate p-values, overall gene ontology assignments and a descriptor of overexpression ('induced') or underexpression ('repressed') normalised to benign specimens from GSE3325. Laser capture microdissected material is subgrouped as described in the original publication and in the methods section incorporating prostatic intraepithelial neoplasia (PIN), localised prostate cancer (PCA), hormone refractory (HR), metastatic hormone refractory (MET_HR) and metastatic hormone naïve disease (MET_HN). In addition gene signatures are defined as sub-signatures of larger signatures generated using lower stringencies/broader branching thresholds, the relationship between gene signatures at different thresholds is indicated in columns X-AE. The number of genes within each coexpression module is given in the column entitled 'moduleSize. varambally'. The numbers of corresponding mapped genes in the other data sets are indicated in columns $Y$ and W. Gene ontology assignments for each signature are provided in column AF.

Additional file 11: Table S9. Gene composition and ontology assignments to statistically significant gene signatures. Sheet one lists the gene signatures and the genes that comprise them which are capable of clustering sample groups within the Tomlins and Ramaswamy datasets (Fischer's exact test $<0.05 \mathrm{p}$-value). Sheet two lists the $\mathrm{GO}$ terms associated with each significant discriminatory gene signature ( $<0.05 p$-value). The 
discriminatory groups shown here mirror those depicted in the heatmap in Figure 1. Sheet three summarises the $\mathrm{GO}$ terms that are significant discriminators of important clinical sample groupings within the Tomlins and Ramaswamy datasets.

Additional file 12: Table S10. A 71-gene signature capable of subclustering localised prostate cancer cases across multiple datasets.

Additional file 13: Table S11. Overexpressed genes in localised prostate cancer versus benign tissue within the entire set of differentially expressed genes in the Varambally dataset (GSE3325).

Additional file 14: Table S12. AUC values for individual probes for ERG, $K L K 3, A R$ and the gene signature in the test dataset from Grasso et al.

Additional file 15: Table S13. AUC values for individual probes for ERG, KLK3, AR and the gene signature in the evaluation dataset from Grasso et al.

\section{Competing interests}

The authors declare that they have no competing interests.

\section{Authors' contributions}

IGM conceived the study and wrote the paper. PE implemented the Ward agglomerative method and analysed the Varambally, Tomlins and Ramaswamy datasets and also wrote the paper. SJB analysed the Taylor and TCGA datasets and wrote the paper. VZ evaluated the performance of the gene signature in the Grasso dataset. All authors read and approved the final manuscript.

\section{Acknowledgements}

I.G.M. is supported by funding from the Norwegian Research Council, Helse Sor-Ost and the University of Oslo through the Centre for Molecular Medicine (Norway), which is the part of the Nordic EMBL (European Molecular Biology Laboratory) partnership and also supported by Oslo University Hospitals. I.G.M. is also supported by the Norwegian Cancer Society and by EU FP7 funding. I.G.M. holds a visiting scientist position with Cancer Research UK through the Cambridge Research Institute and a Senior Visiting Research Fellowship with Cambridge University through the Department of Oncology. SJ.B is funded by the Norwegian Cancer Society and Molecular Life Sciences at the University of Oslo. V. Z. is support by the Centre for Molecular Medicine (Norway) and the Psychosis Research Centre at the Institute for Clinical Medicine at Oslo university Hospitals. P. E. is supported by Cancer Research UK.

\section{Author details}

'Bioinformatics \& Biostatistics, Cancer Research UK London Research Institute, 44 Lincoln's Inn Fields, London WC2A 3PX, UK. Prostate Cancer Research Group, Centre for Molecular Medicine Norway (NCMM), Nordic EMBL Partnership University of Oslo and Oslo University Hospital, Oslo, Norway. ${ }^{3}$ Department of Cancer Prevention and Urology, Institute of Cancer Research and Oslo University Hospital, Oslo, Norway.

Received: 21 August 2014 Accepted: 17 December 2014

Published online: 31 December 2014

\section{References}

1. Rhodes DR, Yu J, Shanker K, Deshpande N, Varambally R, Ghosh D, Barrette T, Pandey A, Chinnaiyan AM: Large-scale meta-analysis of cancer microarray data identifies common transcriptional profiles of neoplastic transformation and progression. Proc Natl Acad Sci U S A 2004, 101:9309-9314.

2. Segal E, Friedman N, Koller D, Regev A: A module map showing conditional activity of expression modules in cancer. Nat Genet 2004, 36:1090-1098.

3. Horvath S, Zhang B, Carlson M, Lu KV, Zhu S, Felciano RM, Laurance MF, Zhao W, Qi S, Chen Z, Lee Y, Scheck AC, Liau LM, Wu H, Geschwind DH, Febbo PG, Kornblum HI, Cloughesy TF, Nelson SF, Mischel PS: Analysis of oncogenic signaling networks in glioblastoma identifies ASPM as a molecular target. Proc Natl Acad Sci U S A 2006, 103:17402-17407.

4. Stuart RO, Wachsman W, Berry CC, Wang-Rodriguez J, Wasserman L, Klacansky I, Masys D, Arden K, Goodison S, McClelland M, Wang Y, Sawyers A, Kalcheva I, Tarin D, Mercola D: In silico dissection of cell-type-associated patterns of gene expression in prostate cancer. Proc Natl Acad Sci USA 2004, 101:615-620.
5. Tomlins SA, Mehra R, Rhodes DR, Cao X, Wang L, Dhanasekaran SM, Kalyana-Sundaram S, Wei JT, Rubin MA, Pienta KJ, Shah RB, Chinnaiyan AM: Integrative molecular concept modeling of prostate cancer progression. Nat Genet 2007, 39:41-51.

6. Ramaswamy S, Ross KN, Lander ES, Golub TR: A molecular signature of metastasis in primary solid tumors. Nat Genet 2003, 33:49-54.

7. Hoerl AE, Kennard RW: Ridge regression: biased estimation for nonorthogonal problems. Technometrics 1970, 12:55-67.

8. Varambally S, Yu J, Laxman B, Rhodes DR, Mehra R, Tomlins SA, Shah RB, Chandran U, Monzon FA, Becich MJ, Wei JT, Pienta KJ, Ghosh D, Rubin MA Chinnaiyan AM: Integrative genomic and proteomic analysis of prostate cancer reveals signatures of metastatic progression. Cancer Cell 2005, 8:393-406.

9. Ward $\mathrm{JH} \mathrm{Jr}$ : Hierarchical grouping to optimize an objective function. $J \mathrm{Am}$ Stat Assoc 1963, 58:236-244.

10. McQuitty LL: Similarity analysis by reciprocal pairs for discrete and continuous data. Educ Psychol Measure 1966, 26:825-831.

11. Shannon CE: A mathematical theory of communication. Bell Syst Tech J 1948, 27:379-423.

12. Chen B-S, Li C-W: On the interplay between entropy and robustness of gene regulatory networks. Entropy 2010, 12:1071-1101.

13. Furlanello C, Serafini M, Merler S, Jurman G: Entropy-based gene ranking without selection bias for the predictive classification of microarray data. BMC Bioinformatics 2003, 4:54.

14. Cuzick J, Swanson GP, Fisher G, Brothman AR, Berney DM, Reid JE, Mesher D, Speights VO, Stankiewicz E, Foster CS, Moller H, Scardino P, Warren JD, Park J, Younus A, Flake DD 2nd, Wagner S, Gutin A, Lanchbury JS, Stone S, Transatlantic Prostate G: Prognostic value of an RNA expression signature derived from cell cycle proliferation genes in patients with prostate cancer: a retrospective study. Lancet Oncol 2011, 12:245-255.

15. Fukuma Y, Matsui H, Koike H, Sekine Y, Shechter I, Ohtake N, Nakata S, Ito K, Suzuki K: Role of squalene synthase in prostate cancer risk and the biological aggressiveness of human prostate cancer. Prostate Cancer Prostatic Dis 2012, 15:339-345.

16. Cuzick J, Berney DM, Fisher G, Mesher D, Moller H, Reid JE, Perry M, Park J, Younus A, Gutin A, Foster CS, Scardino P, Lanchbury JS, Stone S: Prognostic value of a cell cycle progression signature for prostate cancer death in a conservatively managed needle biopsy cohort. Br J Cancer 2012, 106:1095-1099.

17. Yuan X, Cai C, Chen S, Yu Z, Balk SP: Androgen receptor functions in castration-resistant prostate cancer and mechanisms of resistance to new agents targeting the androgen axis. Oncogene 2014, 33:2815-2825.

18. Jenkins RB, Qian J, Lieber MM, Bostwick DG: Detection of c-myc oncogene amplification and chromosomal anomalies in metastatic prostatic carcinoma by fluorescence in situ hybridization. Cancer Res 1997, 57:524-531.

19. Ellwood-Yen K, Graeber TG, Wongvipat J, Iruela-Arispe ML, Zhang J, Matusik $\mathrm{R}$, Thomas GV, Sawyers CL: Myc-driven murine prostate cancer shares molecular features with human prostate tumors. Cancer Cell 2003, 4:223-238.

20. Mikaelsson E, Danesh-Manesh AH, Luppert A, Jeddi-Tehrani M, Rezvany MR, Sharifian RA, Safaie R, Roohi A, Osterborg A, Shokri F, Mellstedt H, Rabbani $\mathrm{H}$ : Fibromodulin, an extracellular matrix protein: characterization of its unique gene and protein expression in B-cell chronic lymphocytic leukemia and mantle cell lymphoma. Blood 2005, 105:4828-4835.

21. Vallat $L$, Magdelenat $H$, Merle-Beral $H$, Masdehors P, Potocki De Montalk G, Davi F, Kruhoffer M, Sabatier L, Orntoft TF, Delic J: The resistance of B-CLL cells to DNA damage-induced apoptosis defined by DNA microarrays. Blood 2003, 101:4598-4606.

22. Levens E, Luo X, Ding L, Williams RS, Chegini N: Fibromodulin is expressed in leiomyoma and myometrium and regulated by gonadotropinreleasing hormone analogue therapy and TGF-beta through Smad and MAPK-mediated signalling. Mol Hum Reprod 2005, 11:489-494.

23. Di Vizio D, Morello M, Sotgia F, Pestell RG, Freeman MR, Lisanti MP: An absence of stromal caveolin-1 is associated with advanced prostate cancer, metastatic disease and epithelial Akt activation. Cell Cycle 2009, 8:2420-2424.

24. Taylor BS, Schultz N, Hieronymus H, Gopalan A, Xiao Y, Carver BS, Arora VK, Kaushik P, Cerami E, Reva B, Antipin Y, Mitsiades N, Landers T, Dolgalev I, Major JE, Wilson M, Socci ND, Lash AE, Heguy A, Eastham JA, Scher HI, Reuter VE, Scardino PT, Sander C, Sawyers CL, Gerald WL: Integrative genomic profiling of human prostate cancer. Cancer Cell 2010, 18:11-22. 
25. Rhodes DR, Yu J, Shanker K, Deshpande N, Varambally R, Ghosh D, Barrette T, Pandey A, Chinnaiyan AM: ONCOMINE: a cancer microarray database and integrated data-mining platform. Neoplasia 2004, 6:1-6.

26. Itkonen HM, Engedal N, Babaie E, Luhr M, Guldvik IJ, Minner S, Hohloch J, Tsourlakis MC, Schlomm T, Mills IG: UAP1 is overexpressed in prostate cancer and is protective against inhibitors of $\mathrm{N}$-linked glycosylation. Oncogene 2014. Advance online publication 22nd September; doi: 10.1038/ onc.2014.307

27. Hagisawa S, Ohyama C, Takahashi T, Endoh M, Moriya T, Nakayama J, Arai Y, Fukuda M: Expression of core 2 beta1,6-N-acetylglucosaminyltransferase facilitates prostate cancer progression. Glycobiology 2005, 15:1016-1024.

28. Ma W, Diep K, Fritsche HA, Shore N, Albitar M: Diagnostic and prognostic scoring system for prostate cancer using urine and plasma biomarkers. Genet Test Mol Biomarkers 2014, 18:156-163.

29. Kamigaito T, Okaneya T, Kawakubo M, Shimojo H, Nishizawa O, Nakayama J: Overexpression of O-GICNAc by prostate cancer cells is significantly associated with poor prognosis of patients. Prostate Cancer Prostatic Dis 2014, 17:18-22.

30. Itkonen HM, Minner S, Guldvik IJ, Sandmann MJ, Tsourlakis MC, Berge V, Svindland A, Schlomm T, Mills IG: O-GlcNAc transferase integrates metabolic pathways to regulate the stability of c-MYC in human prostate cancer cells. Cancer Res 2013, 73:5277-5287.

31. Gurel B, Iwata T, Koh CM, Jenkins RB, Lan F, Van Dang C, Hicks JL, Morgan J, Cornish TC, Sutcliffe S, Isaacs WB, Luo J, De Marzo AM: Nuclear MYC protein overexpression is an early alteration in human prostate carcinogenesis. Mod Pathol 2008, 21:1156-1167.

32. Chou TY, Hart GW, Dang CV: c-Myc is glycosylated at threonine 58, a known phosphorylation site and a mutational hot spot in lymphomas. J Biol Chem 1995, 270:18961-18965.

33. Tomlins SA, Rhodes DR, Perner S, Dhanasekaran SM, Mehra R, Sun XW, Varambally S, Cao X, Tchinda J, Kuefer R, Lee C, Montie JE, Shah RB, Pienta $\mathrm{KJ}$, Rubin MA, Chinnaiyan AM: Recurrent fusion of TMPRSS2 and ETS transcription factor genes in prostate cancer. Science 2005, 310:644-648.

34. Deyoung MP, Scheurle D, Damania H, Zylberberg C, Narayanan R: Down's syndrome-associated single minded gene as a novel tumor marker. Anticancer Res 2002, 22:3149-3157.

35. Halvorsen OJ, Rostad K, Oyan AM, Puntervoll H, Bo TH, Stordrange L, Olsen S, Haukaas SA, Hood L, Jonassen I, Kalland KH, Akslen LA: Increased expression of SIM2-s protein is a novel marker of aggressive prostate cancer. Clin Cancer Res 2007, 13:892-897.

36. Koh CM, Gurel B, Sutcliffe S, Aryee MJ, Schultz D, Iwata T, Uemura M, Zeller KI, Anele U, Zheng Q, Hicks JL, Nelson WG, Dang CV, Yegnasubramanian S, De Marzo AM: Alterations in nucleolar structure and gene expression programs in prostatic neoplasia are driven by the MYC oncogene. Am J Pathol 2011, 178:1824-1834.

37. Wang L, Li Y, Yang X, Yuan H, Li X, Qi M, Chang YW, Wang C, Fu W, Yang M, Zhang J, Han B: ERG-SOX4 interaction promotes epithelial-mesenchymal transition in prostate cancer cells. Prostate 2014, 74:647-658.

38. Leshem O, Madar S, Kogan-Sakin I, Kamer I, Goldstein I, Brosh R, Cohen Y, Jacob-Hirsch J, Ehrlich M, Ben-Sasson S, Goldfinger N, Loewenthal R, Gazit E, Rotter $\mathrm{V}$, Berger R: TMPRSS2/ERG promotes epithelial to mesenchymal transition through the ZEB1/ZEB2 axis in a prostate cancer model. PLOS One 2011, 6:e21650.

39. Ribeiro FR, Paulo P, Costa VL, Barros-Silva JD, Ramalho-Carvalho J, Jeronimo C, Henrique R, Lind GE, Skotheim RI, Lothe RA, Teixeira MR: Cysteine-rich secretory protein-3 (CRISP3) is strongly up-regulated in prostate carcinomas with the TMPRSS2-ERG fusion gene. PLoS One 2011, 6:e22317.

40. Itkonen HM, Mills IG: N-linked glycosylation supports cross-talk between receptor tyrosine kinases and androgen receptor. PLoS One 2013, 8:e65016.

41. Visakorpi T, Hyytinen E, Koivisto P, Tanner M, Keinanen R, Palmberg C, Palotie A, Tammela T, Isola J, Kallioniemi OP: In vivo amplification of the androgen receptor gene and progression of human prostate cancer. Nat Genet 1995, 9:401-406.

42. Tomlins SA, Aubin SM, Siddiqui J, Lonigro RJ, Sefton-Miller L, Miick S, Williamsen S, Hodge P, Meinke J, Blase A, Penabella Y, Day JR, Varambally R, Han B, Wood D, Wang L, Sanda MG, Rubin MA, Rhodes DR, Hollenbeck B, Sakamoto K, Silberstein JL, Fradet $Y$, Amberson JB, Meyers S, Palanisamy N, Rittenhouse H, Wei JT, Groskopf J, Chinnaiyan AM: Urine TMPRSS2:ERG fusion transcript stratifies prostate cancer risk in men with elevated serum PSA. Sci Transl Med 2011, 3:94ra72.
43. Grasso CS, Wu YM, Robinson DR, Cao X, Dhanasekaran SM, Khan AP, Quist MJ, Jing X, Lonigro RJ, Brenner JC, Asangani IA, Ateeq B, Chun SY, Siddiqui J, Sam L, Anstett M, Mehra R, Prensner JR, Palanisamy N, Ryslik GA, Vandin F, Raphael BJ, Kunju LP, Rhodes DR, Pienta KJ, Chinnaiyan AM, Tomlins SA: The mutational landscape of lethal castration-resistant prostate cancer. Nature 2012, 487:239-243.

44. Fleischmann A, Saramaki OR, Zlobec I, Rotzer D, Genitsch V, Seiler R, Visakorpi T, Thalmann GN: Prevalence and prognostic significance of TMPRSS2-ERG gene fusion in lymph node positive prostate cancers. Prostate 2014, 74:1647-1654.

45. Nam RK, Sugar L, Wang Z, Yang W, Kitching R, Klotz LH, Venkateswaran V, Narod SA, Seth A: Expression of TMPRSS2:ERG gene fusion in prostate cancer cells is an important prognostic factor for cancer progression. Cancer Biol Ther 2007, 6:40-45.

46. Saramaki OR, Harjula AE, Martikainen PM, Vessella RL, Tammela TL, Visakorp T: TMPRSS2:ERG fusion identifies a subgroup of prostate cancers with a favorable prognosis. Clin Cancer Res 2008, 14:3395-3400.

\section{Submit your next manuscript to BioMed Central and take full advantage of:}

- Convenient online submission

- Thorough peer review

- No space constraints or color figure charges

- Immediate publication on acceptance

- Inclusion in PubMed, CAS, Scopus and Google Scholar

- Research which is freely available for redistribution

Submit your manuscript at www.biomedcentral.com/submit
C Biomed Central 